ANALYSIS \& PDE

Volume $2 \quad$ No. $1 \quad 2009$

\author{
ENNO LENZMANN
}

UNIQUENESS OF GROUND STATES

FOR PSEUDORELATIVISTIC HARTREE EQUATIONS 


\title{
UNIQUENESS OF GROUND STATES FOR PSEUDORELATIVISTIC HARTREE EQUATIONS
}

\author{
ENNO LENZMANN
}

We prove uniqueness of ground states $Q \in H^{1 / 2}\left(\mathbb{R}^{3}\right)$ for the pseudorelativistic Hartree equation,

$$
\sqrt{-\Delta+m^{2}} Q-\left(|x|^{-1} *|Q|^{2}\right) Q=-\mu Q,
$$

in the regime of $Q$ with sufficiently small $L^{2}$-mass. This result shows that a uniqueness conjecture by Lieb and Yau [1987] holds true at least for $N=\int|Q|^{2} \ll 1$ except for at most countably many $N$.

Our proof combines variational arguments with a nonrelativistic limit, leading to a certain Hartreetype equation (also known as the Choquard-Pekard or Schrödinger-Newton equation). Uniqueness of ground states for this limiting Hartree equation is well-known. Here, as a key ingredient, we prove the socalled nondegeneracy of its linearization. This nondegeneracy result is also of independent interest, for it proves a key spectral assumption in a series of papers on effective solitary wave motion and classical limits for nonrelativistic Hartree equations.

\section{Introduction}

The pseudorelativistic Hartree energy functional, given (in appropriate units) by

$$
\mathscr{E}(\psi)=\int_{\mathbb{R}^{3}} \bar{\psi} \sqrt{-\Delta+m^{2}} \psi-\frac{1}{2} \int_{\mathbb{R}^{3}}\left(|x|^{-1} *|\psi|^{2}\right)|\psi|^{2},
$$

arises in the mean-field limit of a quantum system describing many self-gravitating, relativistic bosons with rest mass $m>0$. Such a physical system is often referred to as a boson star, and various models for these - at least theoretical — objects have attracted a great deal of attention in theoretical and numerical astrophysics over the past years.

In order to gain some rigorous insight into the theory of boson stars, it is of particular interest to study ground states (that is, minimizers) for the variational problem

$$
E(N)=\inf \left\{\mathscr{E}(\psi): \psi \in H^{1 / 2}\left(\mathbb{R}^{3}\right) \text { and } \int_{\mathbb{R}^{3}}|\psi|^{2}=N\right\},
$$

where the parameter $N>0$ plays the role of the stellar mass. Provided that problem (1-2) has indeed a ground state $Q \in H^{1 / 2}\left(\mathbb{R}^{3}\right)$, one readily finds that it satisfies the pseudorelativistic Hartree equation,

$$
\sqrt{-\Delta+m^{2}} Q-\left(|x|^{-1} *|Q|^{2}\right) Q=-\mu Q,
$$

with $\mu=\mu(Q) \in \mathbb{R}$ being some Lagrange multiplier.

MSC2000: 35Q55.

Keywords: pseudorelativistic Hartree equation, ground state, uniqueness, boson stars.

Partly supported by NSF Grant DMS-0702492. 
In fact, the existence of symmetric-decreasing ground states $Q=Q^{*}(|x|) \geq 0$ minimizing (1-2) was first proven by Lieb and Yau [1987], where the authors also conjectured that uniqueness holds true in the following sense. For each $N>0$, the variational problem (1-2) has at most one symmetric-decreasing ground state. If true, this result further implies, by strict rearrangement inequalities, that we have indeed uniqueness of all the ground states of (1-2) for each $N>0$, up to phase and translation.

However, the nonlocality of $\sqrt{-\Delta+m^{2}}$ as well as the convolution-type nonlinearity both complicate the analysis of the pseudorelativistic Hartree equation (1-3) in a substantial way. In particular, the set of its radial solutions is not amenable to ODE techniques (for example, shooting arguments and comparison principles) which are key arguments for proving uniqueness of ground states for nonlinear Schrödinger equations (NLS) with local nonlinearities; see [Peletier and Serrin 1983; McLeod and Serrin 1987; Kwong 1989; McLeod 1993].

A further complication in the analysis of (1-3) stems from the fact that there are no simple scaling arguments that relate ground states with different $N$, due to the presence of $m>0$. Indeed, this lack of a simple scaling mechanism is essential for the existence of a critical stellar mass $N_{*}>0$; see Theorem 1.

As a first step towards proving uniqueness of ground states for (1-2), we present Theorem 2 below, which shows that ground states for problem are indeed unique (modulo translation and phase) for all sufficiently small $N>0$ except for at most countably many. Our proof uses variational arguments combined with a nonrelativistic limit, leading to the nonlinear Hartree equation (also called ChoquardPekar or Schrödinger-Newton equation) given by

$$
-\frac{1}{2 m} \Delta Q_{\infty}-\left(|x|^{-1} *\left|Q_{\infty}\right|^{2}\right) Q_{\infty}=-\lambda Q_{\infty}
$$

It is known this equation has a unique radial, positive solution $Q_{\infty} \in H^{1}\left(\mathbb{R}^{3}\right)$ for $\lambda>0$ given; see [Lieb 1977] and Appendix A.

In the present paper, we prove (as a key ingredient) that $Q_{\infty} \in H^{1}\left(\mathbb{R}^{3}\right)$ has a nondegenerate linearization. By this we mean that the linearization of (1-4) around $Q_{\infty}$ has a nullspace that is entirely due to the equation's invariance under phase and translation transformation; see Theorem 4 below and its remarks for a precise statement. In particular, we show that the linear operator $L_{+}$given by

$$
L_{+} \xi=-\frac{1}{2 m} \Delta \xi+\lambda \xi-\left(|x|^{-1} *\left|Q_{\infty}\right|^{2}\right) \xi-2 Q_{\infty}\left(|x|^{-1} *\left(Q_{\infty} \xi\right)\right)
$$

satisfies

$$
\operatorname{ker} L_{+}=\operatorname{span}\left\{\partial_{x_{1}} Q_{\infty}, \partial_{x_{2}} Q_{\infty}, \partial_{x_{3}} Q_{\infty}\right\} .
$$

Furthermore, by a perturbation argument, we conclude an analogous nondegeneracy result for ground states of the pseudorelativistic Hartree equation (1-3) with sufficiently small $L^{2}$-mass; see Theorem 3 below.

In addition to being a mere technical key fact proven in this paper, the nondegeneracy result for (1-4) is also of independent interest. For example, it proves a key spectral assumption in a series of papers on effective solitary wave motion and classical limits for Hartree equations; see [Fröhlich et al. 2002; 2004; Jonsson et al. 2006; Abou Salem 2007] and also the remark following Theorem 4. Another very recent application of the nondegeneracy result (1-6) is presented in [Krieger et al. 2008], where two soliton solutions to the time-dependent version of (1-4) are constructed. 
In the context of ground states for NLS with local nonlinearities, the nondegeneracy of linearizations is a well-known fact (see [Weinstein 1985; Chang et al. 2007]) and it plays a central role in the stability analysis of solitary waves for NLS. However, the arguments for NLS with local nonlinearities make use of Sturm-Liouville theory, which, by contrast, is not applicable to $L_{+}$given by (1-5) due to its nonlocal character. For more details, we refer to Section 7 below.

Apart from their minimizing property, the ground states for (1-2) also play an important role for the time-dependent pseudorelativistic Hartree equation,

$$
i \partial_{t} \psi=\sqrt{-\Delta+m^{2}} \psi-\left(|x|^{-1} *|\psi|^{2}\right) \psi,
$$

with the wave field $\psi:[0, T) \times \mathbb{R}^{3} \rightarrow \mathbb{C}$. Clearly, Equation (1-7) has solitary wave solutions

$$
\psi(t, x)=e^{i t \mu} Q(x),
$$

whenever $Q \in H^{1 / 2}\left(\mathbb{R}^{3}\right)$ is a nontrivial solution to (1-3). Let us also mention that the dispersive nonlinear PDE (1-7) exhibits a rich variety of phenomena, such as stable and unstable traveling solitary waves, as well as finite-time blowup solutions indicating the "gravitational collapse" of a boson star; see [Fröhlich et al. 2007a; 2007b; Fröhlich and Lenzmann 2007]. For well-posedness results concerning (1-7) and its rigorous derivation from many-body quantum mechanics, we refer to [Cho and Ozawa 2006; Lenzmann 2007] and [Elgart and Schlein 2007], respectively.

For the reader's convenience, we conclude our introduction by summarizing the existence result about ground states for problem (1-2) along with a list of their basic properties.

Theorem 1 (Existence and properties of ground states). Suppose that $m>0$ holds in (1-1). Then there exists a universal constant $N_{*}>4 / \pi$ (independent of $m$ ) such that the following holds.

(i) (Existence) There exists a ground state $Q \in H^{1 / 2}\left(\mathbb{R}^{3}\right)$ for problem (1-2) if and only if

$$
0<N<N_{*} .
$$

Moreover, the function $Q$ satisfies the pseudorelativistic Hartree equation (1-3) in the sense of distributions with some Lagrange multiplier $\mu \in \mathbb{R}$.

(ii) (Smoothness and exponential decay) Any ground state $Q$ belongs to $H^{s}\left(\mathbb{R}^{d}\right)$ for all $s \geq 0$ and $e^{+\delta|x|} Q \in L^{\infty}\left(\mathbb{R}^{3}\right)$ for some $\delta=\delta(Q)>0$.

(iii) (Radiality and strict positivity) Any ground state $Q$ is equal to its spherical-symmetric rearrangement $Q^{*}(|x|)$ up to phase and translation. Moreover, we have $Q^{*}(|x|)>0$ for all $x \in \mathbb{R}^{3}$.

Remark. For the proofs of (i) and (ii)-(iii), we refer to [Lieb and Yau 1987] and [Lenzmann 2006; Fröhlich et al. 2007a], respectively. In physical terms, the constant $N_{*}>0$ can be regarded as the "Chandrasekhar limit mass" of a pseudorelativistic boson star.

\section{Main results}

We now state our first main result concerning the uniqueness of ground states for the pseudorelativistic Hartree equation (1-3). 
Theorem 2 (Uniqueness of ground states for $N \ll 1$ ). Assume that $m>0$ holds in (1-1). Then, for $0<N \ll 1$, we have uniqueness of ground states for problem (1-2) up to phase and translation whenever $E^{\prime}(N)$ exists. In particular, the symmetric-decreasing ground state $Q=Q^{*} \in H^{1 / 2}\left(\mathbb{R}^{3}\right)$ minimizing (1-2) is unique for such $N>0$.

Remarks. (1) Since it is known from [Lieb and Yau 1987] that the ground state energy $E(N)$ is strictly concave, the derivative $E^{\prime}(N)$ exists for all $N \in\left(0, N_{*}\right)$, except on a subset $\Sigma$ which is at most countable. In particular, it is easy to see that the Lagrange multiplier $\mu$ is unique for such $N \in\left(0, N_{*}\right) \backslash \Sigma$, in the sense that $\mu$ only depends on $Q$ through $N=\int|Q|^{2}$. Our argument to prove Theorem 2 has to avoid the "exceptional" set $\Sigma$. A natural conjecture would be that $\Sigma=\varnothing$ holds.

(2) It would be desirable to extend this uniqueness result (whose proof partly relies on perturbative arguments) to the whole range $0<N<N_{*}$ of existence; or, more interestingly, to disprove uniqueness for some $N>0$ sufficiently large.

(3) By definition, ground states for the pseudorelativistic Hartree equation (1-2) are always minimizers for the variational problem (1-2). In principle, we cannot exclude the possibility that (1-3) has a positive solution without being a minimizer for (1-2).

(4) To the author's knowledge, this is the first uniqueness result for ground states that solve a nonlinear pseudo-differential equation in space dimension $n>1$. In fact, apart from a very special case arising in $n=1$ dimensions for solitary waves solving Benjamin-Ono-type equations (see [Amick and Toland 1991; Albert 1995]), nothing seems to be known, for instance, about uniqueness of ground states $\varphi \in H^{s}\left(\mathbb{R}^{n}\right)$ for nonlinear equations involving the fractional Laplacian $(-\Delta)^{s / 2} \varphi+f(\varphi)=-\mu \varphi$, where $f(\varphi)$ denotes some nonlinearity and $\mu \in \mathbb{R}$ is given. The author plans to pursue this question in future work.

(5) If $m=0$ vanishes, we have existence of ground states for problem (1-2) if and only if $N=N_{*}$. In what follows, we shall exclusively deal with the physically relevant case where $m>0$ holds. Nevertheless, it remains an interesting open question whether uniqueness of ground states also holds for $m=0$, since the methods developed here are clearly not applicable to this limiting case.

Our next result proves a so-called nondegeneracy condition, which was introduced in [Fröhlich et al. $2007 \mathrm{~b}]$ as a spectral assumption supported by numerical evidence. There, the effective motion of solitary waves for (1-7) with an slowly varying external potential was studied. Furthermore, the following nondegeneracy result allows us to give an unconditional proof for the cylindrical symmetry of traveling solitary waves for the time-dependent pseudorelativistic Hartree equation (1-7); see [Fröhlich et al. 2007b] for more details. The precise nondegeneracy statement reads as follows.

Theorem 3 (Nondegeneracy of ground states for $N \ll 1$ ). Let $m>0$ in (1-1) and suppose that $Q=Q^{*}$ is a symmetric-decreasing ground state for problem (1-2) with Lagrange multiplier $\mu \in \mathbb{R}$. Furthermore, we consider the linear operator $L_{+}$given by

$$
L_{+} \xi=\left(\sqrt{-\Delta+m^{2}}+\mu\right) \xi-\left(|x|^{-1} *|Q|^{2}\right) \xi-2 Q\left(|x|^{-1} *(Q \xi)\right),
$$

acting on $L^{2}\left(\mathbb{R}^{3}\right)$ with domain $H^{1}\left(\mathbb{R}^{3}\right)$. Then, for $0<N \ll 1$, the operator $L_{+}$is nondegenerate, that is, its kernel satisfies

$$
\operatorname{ker} L_{+}=\operatorname{span}\left\{\partial_{x_{1}} Q, \partial_{x_{2}} Q, \partial_{x_{3}} Q\right\}
$$


Remarks. (1) This completely characterizes the kernel of the linearization of the pseudorelativistic Hartree equation (1-3) around ground state $Q=Q^{*}$ with $\int|Q|^{2} \ll 1$. Note that, due to the presence of $|Q|^{2}$ in the nonlinearity, the linearized operator is not $\mathbb{C}$-linear. See also the remark following Theorem 4 below for more details on the analogous statement for the nonrelativistic equation (1-4).

(2) The nondegeneracy of $L_{+}$holds for all $N=\int|Q|^{2} \ll 1$. The extra condition that $E^{\prime}(N)$ exists, which is present in Theorem 2, is not needed here.

In order to prove Theorem 3, we first have to show the nondegeneracy for the linearization around the ground state $Q_{\infty} \in H^{1}\left(\mathbb{R}^{3}\right)$ solving the nonrelativistic Hartree equation (1-4). As mentioned before, this spectral result is of independent interest, since it proves a key assumption in [Fröhlich et al. 2002; Fröhlich et al. 2004; Jonsson et al. 2006; Abou Salem 2007]. See also [Krieger et al. 2008], where the following nondegeneracy result is needed. Hence we record this fact about (1-4) as one of our main results.

Theorem 4 (Nondegeneracy for $Q_{\infty}$ ). Let $m>0$ and $\lambda>0$ be given. Furthermore, suppose that $Q_{\infty} \in H^{1}\left(\mathbb{R}^{3}\right)$ is the unique radial, positive solution to the nonrelativistic Hartree equation (1-4). Then the linear operator $L_{+}$given by

$$
L_{+} \xi=-\frac{1}{2 m} \Delta \xi+\lambda \xi-\left(|x|^{-1} *\left|Q_{\infty}\right|^{2}\right) \xi-2 Q_{\infty}\left(|x|^{-1} *\left(Q_{\infty} \xi\right)\right)
$$

acting on $L^{2}\left(\mathbb{R}^{3}\right)$ with domain $H^{2}\left(\mathbb{R}^{3}\right)$, satisfies

$$
\operatorname{ker} L_{+}=\operatorname{span}\left\{\partial_{x_{1}} Q_{\infty}, \partial_{x_{2}} Q_{\infty}, \partial_{x_{3}} Q_{\infty}\right\} .
$$

Remarks. (1) The linearized operator $L$ for (1-4) at $Q_{\infty}$ is found to be

$$
L h=-\frac{1}{2 m} \Delta h+\lambda h-\left(|x|^{-1} *\left|Q_{\infty}\right|^{2}\right) h-Q_{\infty}\left(|x|^{-1} *\left(Q_{\infty}(h+\bar{h})\right)\right) .
$$

It is convenient to view the operator $L$ (which is not $\mathbb{C}$-linear) as acting on

$$
\left(\begin{array}{c}
\operatorname{Re} h \\
\operatorname{Im} h
\end{array}\right)
$$

so that it can be written as

$$
L=\left(\begin{array}{ll}
L_{+} & 0 \\
0 & L_{-}
\end{array}\right) .
$$

Here $L_{+}$is as in Theorem 4 above, and $L_{-}$is the (local) operator

$$
L_{-}=-\frac{1}{2 m} \Delta+\lambda-\left(|x|^{-1} *\left|Q_{\infty}\right|^{2}\right) .
$$

It is easy to see that $\operatorname{ker} L_{-}=\operatorname{span}\left\{Q_{\infty}\right\}$ holds. Hence, by Theorem 4, we obtain

$$
\operatorname{ker} L=\operatorname{span}\left\{\left(\begin{array}{c}
\partial_{x_{1}} Q_{\infty} \\
0
\end{array}\right),\left(\begin{array}{c}
\partial_{x_{2}} Q_{\infty} \\
0
\end{array}\right),\left(\begin{array}{c}
\partial_{x_{2}} Q_{\infty} \\
0
\end{array}\right),\left(\begin{array}{c}
0 \\
Q_{\infty}
\end{array}\right)\right\} .
$$

(2) The precise knowledge of $\operatorname{ker} L$ implies, by well-known arguments along the lines for NLS with local nonlinearities (given in [Weinstein 1985]), the following coercivity estimate: There is a constant 
$\delta>0$ such that

$$
\left\langle f, L_{+} f\right\rangle+\left\langle g, L_{-} g\right\rangle \geq \delta\left(\|f\|_{H^{1}}^{2}+\|g\|_{H^{1}}^{2}\right),
$$

when $f \perp \operatorname{span}\left\{Q_{\infty}, x_{i} Q_{\infty}\right\}_{i=1}^{3}$ and $g \perp$ span $\left\{2 Q_{\infty}+r \partial_{r} Q_{\infty}, \partial_{x_{i}} Q_{\infty}\right\}_{i=1}^{3}$, which means that $(f, g)$ is symplectically orthogonal to the "soliton manifold" generated by $Q_{\infty}$; see, for example, [Fröhlich et al. 2004]. This coercivity estimate plays a central role in the stability analysis of solitary waves for NLStype equations and their effective motion in an external potential; see, for example, [Weinstein 1985; Bronski and Jerrard 2000; Fröhlich et al. 2004; 2007b; Jonsson et al. 2006; Abou Salem 2007; Holmer and Zworski 2008].

Organization of the paper. This paper is structured as follows. In Section 3, we study the nonrelativistic limit of ground states for a dimensionalized version of the variational problem (1-2). In Section 4, we prove a nondegeneracy result for the nonrelativistic ground state $Q_{\infty} \in H^{1}\left(\mathbb{R}^{3}\right)$ in the radial setting. Then, in Section 5, we establish a local uniqueness result around $Q_{\infty} \in H^{1}\left(\mathbb{R}^{3}\right)$ by means of an implicitfunction-type argument.

We prove Theorem 2 in Section 6, and Theorems 3 and 4 in Section 7. Appendices A and B collect some auxiliary results and we also give a uniqueness proof for the ground state $Q_{\infty} \in H^{1}\left(\mathbb{R}^{3}\right)$, which differs from [Lieb 1977] in certain ways.

Notation and conventions. As usual $H^{s}\left(\mathbb{R}^{n}\right)$ stands for the inhomogeneous Sobolev space of order $s \in \mathbb{R}$, equipped with norm $\|f\|_{H^{s}}=\left\|\langle\nabla\rangle^{s} f\right\|_{L^{2}}$, where $\langle\nabla\rangle$ is defined via its multiplier $\langle\xi\rangle=\left(1+\xi^{2}\right)^{1 / 2}$ in the Fourier domain. Also, we shall make use of the space of radial and real-valued functions that belong to $H^{1}\left(\mathbb{R}^{3}\right)$, which we denote by

$$
H_{\mathrm{r}}^{1}\left(\mathbb{R}^{3}\right)=\left\{f: f \in H^{1}\left(\mathbb{R}^{3}\right), f \text { is radial and real-valued }\right\} .
$$

With the usual abuse of notation we shall write both $f(x)$ and $f(r)$, with $r=|x|$, for radial functions $f$ on $\mathbb{R}^{n}$. For any measurable function $f: \mathbb{R}^{n} \rightarrow \mathbb{C}$ that vanishes at infinity, we denote its symmetric-decreasing rearrangement by $f^{*}=f^{*}(r) \geq 0$.

Throughout this paper, we assume that the mass parameter $m>0$ in (1-1) is strictly positive, which is the physically relevant case.

For the reader's orientation, we mention that our definition of $\mathscr{E}(\psi)$ in (1-1) differs from the conventions in [Lieb and Yau 1987; Fröhlich et al. 2007a] by an inessential factor of 2 and by the fact that we use $\sqrt{-\Delta+m^{2}}$ instead of $\sqrt{-\Delta+m^{2}}-m$. Obviously, these slight alterations in our definition of $\mathscr{E}(\psi)$ do not affect any results on (1-2) that are derived or quoted in the present paper.

Finally, we point out that the function $Q_{\infty} \in H_{r}^{1}\left(\mathbb{R}^{3}\right)$, which denotes the unique ground state for (1-4), appears throughout the paper. However, for the sake of simple notation, we shall also denote all its rescaled copies $a Q_{\infty}(b \cdot)$, with $a>0$ and $b>0$, simply by $Q_{\infty}$, whenever there is no source of confusion.

\section{Nonrelativistic limit}

As a preliminary step towards the proof of Theorems 2 and 3, we study the nonrelativistic limit of ground states for the pseudorelativistic Hartree energy functional. More precisely, we reinstall the speed of light 
$c>0$ into $\mathscr{E}(\psi)$ defined in (1-1), which yields the $c$-depending Hartree energy functional

$$
\mathscr{E}_{c}(\psi)=\int_{\mathbb{R}^{3}} \bar{\psi} \sqrt{-c^{2} \Delta+m^{2} c^{4}} \psi-\frac{1}{2} \int_{\mathbb{R}^{3}}\left(|x|^{-1} *|\psi|^{2}\right)|\psi|^{2} .
$$

An elementary calculation shows that, for any $\psi \in H^{1 / 2}\left(\mathbb{R}^{3}\right)$,

$$
\mathscr{E}(\psi)=c^{-3 \mathscr{E}_{c}}(\tilde{\psi}), \quad \text { with } \psi(x)=c^{-2} \tilde{\psi}\left(c^{-1} x\right) .
$$

Thus we immediately find the following equivalence.

Lemma 1. Let $c>0$ and $N>0$. Then $\widetilde{Q} \in H^{1 / 2}\left(\mathbb{R}^{3}\right)$ minimizes $\mathscr{E}_{c}(\psi)$ subject to $\int|\psi|^{2}=N$ if and only if $Q=c^{-2} \widetilde{Q}\left(c^{-1} \cdot\right)$ minimizes $\mathscr{E}(\psi)$ subject to $\int|\psi|^{2}=c^{-1} N$.

In particular, we have existence of ground states for $\mathscr{E}_{c}(\psi)$ subject to $\int|\psi|^{2}=N$ if and only if $0<N<c N_{*}$ holds, where $N_{*}>4 / \pi$ denotes the same universal constant as in Theorem 1.

We now study the behavior of ground states $Q_{c}$ for $\mathscr{E}_{c}(\psi)$ as $c \rightarrow \infty$ with $\int_{\mathbb{R}^{3}}\left|Q_{c}\right|^{2}=N$ being fixed. By Lemma 1, this is equivalent (after a suitable rescaling) to studying ground states for $\mathscr{E}(\psi)$ with $\int|\psi|^{2}=N$ as $N \rightarrow 0$. However, the following analysis turns out to be more transparent when working with $c>0$ as a parameter and sending $c$ to infinity. Concerning the nonrelativistic limit $c \rightarrow \infty$ of ground states for $\mathscr{E}_{c}(\psi)$, we have the following result.

Proposition 1. Let $m>0$ and $N>0$ be given, and suppose that $c_{n} \rightarrow \infty$ as $n \rightarrow \infty$. Furthermore, we assume that $\left\{Q_{c_{n}}\right\}_{n=1}^{\infty}$ is a sequence of symmetric-decreasing ground states such that $\int_{\mathbb{R}^{3}}\left|Q_{c_{n}}\right|^{2}=N$ for all $n \geq 1$, and each $Q_{c_{n}} \in H^{1 / 2}\left(\mathbb{R}^{3}\right)$ minimizes $\mathscr{E}_{c_{n}}(\psi)$ subject to $\int_{\mathbb{R}^{3}}|\psi|^{2}=N$. Finally, let $\left\{\mu_{c_{n}}\right\}_{n=1}^{\infty}$ denote the sequence of Lagrange multipliers corresponding to $\left\{Q_{c_{n}}\right\}_{n=1}^{\infty}$.

Then the following holds:

$$
\begin{array}{rlrl}
Q_{c_{n}} & \rightarrow Q_{\infty} \quad \text { in } \quad H^{1}\left(\mathbb{R}^{3}\right) \quad \text { as } & n \rightarrow \infty, \\
-\mu_{c_{n}}-m c_{n}^{2} & \rightarrow-\lambda
\end{array}
$$

where $Q_{\infty} \in H^{1}\left(\mathbb{R}^{3}\right)$ is the unique radial, positive solution to

$$
-\frac{1}{2 m} \Delta Q_{\infty}-\left(|x|^{-1} *\left|Q_{\infty}\right|^{2}\right) Q_{\infty}=-\lambda Q_{\infty}
$$

such that $\int_{\mathbb{R}^{3}}\left|Q_{\infty}\right|^{2}=N$. Here $\lambda>0$ is determined through $Q_{\infty}=Q_{\infty}^{*} \in H^{1}\left(\mathbb{R}^{3}\right)$, which is the unique symmetric-decreasing minimizer of the variational problem

$$
E_{\mathrm{nr}}(N)=\inf \left\{\mathscr{E}_{\mathrm{nr}}(\psi): \psi \in H^{1}\left(\mathbb{R}^{3}\right) \text { and } \int_{\mathbb{R}^{3}}|\psi|^{2}=N\right\},
$$

where

$$
\mathscr{E}_{\mathrm{nr}}(\psi)=\frac{1}{2 m} \int_{\mathbb{R}^{3}}|\nabla \psi|^{2}-\frac{1}{2} \int_{\mathbb{R}^{3}}\left(|x|^{-1} *|\psi|^{2}\right)|\psi|^{2} .
$$

Remarks. (1) A similar result for the nonrelativistic limit of ground states (and excited states) solving the Dirac-Fock equations can be found in [Esteban and Séré 2001]. However, unlike the Dirac-Fock and Hartree-Fock energy functionals in atomic physics treated in [Esteban and Séré 2001], the energy functional in (3-1) is not weakly lower semicontinuous due to its attractive potential term. Therefore, an a priori bound on the sequence of Lagrange multipliers $\mu_{c_{n}}$ (away from the essential spectrum of the 
limiting equation) is not sufficient to conclude strong convergence. To deal with this, we also have to use the radial symmetry of the $Q_{c_{n}}$ in order to prove strong convergence.

(2) The uniqueness of the symmetric-decreasing ground state for problem (3-4) was proven by Lieb [1977]. For the reader's convenience, we provide a (partly different) proof of this fact in Appendix A.

3.1. Proof of Proposition 1. We begin with some auxiliary results.

Lemma 2. Let $\left\{\mu_{c_{n}}\right\}_{n=1}^{\infty}$ be as in Proposition 1. Then there exist constants $\delta_{1}>0$ and $\delta_{2}>0$ such that

$$
m c_{n}^{2}-\delta_{1} \leq-\mu_{c_{n}} \leq m c_{n}^{2}-\delta_{2}, \quad \text { for all } n \geq n_{0},
$$

where $n_{0} \gg 1$ is some number.

Proof. The existence of $\delta_{2}>0$ can be deduced as follows. The Euler-Lagrange equation for $Q_{c_{n}}$ reads

$$
\sqrt{-c_{n}^{2} \Delta+m^{2} c_{n}^{4}} Q_{c_{n}}-\left(|x|^{-1} *\left|Q_{c_{n}}\right|^{2}\right) Q_{c_{n}}=-\mu_{c_{n}} Q_{c_{n}},
$$

which upon multiplication with $Q_{c_{n}}$ and integration gives us

$$
\mathscr{\mathscr { E }}_{c_{n}}\left(Q_{c_{n}}\right)-\frac{1}{2} \int_{\mathbb{R}^{3}}\left(|x|^{-1} *\left|Q_{c_{n}}\right|^{2}\right)\left|Q_{c_{n}}\right|^{2}=-\mu_{c_{n}} N .
$$

Next, we recall the operator inequality

$$
\sqrt{-c^{2} \Delta+m^{2} c^{4}} \leq-\frac{1}{2 m} \Delta+m c^{2},
$$

which directly follows in the Fourier domain and the fact that $\sqrt{1+t} \leq t / 2+1$ holds for all $t \geq 0$. Hence we have that $\mathscr{E}_{c_{n}}\left(Q_{c_{n}}\right) \leq \mathscr{E}_{\mathrm{nr}}\left(Q_{c_{n}}\right)+N m c_{n}^{2}$. Furthermore, since $Q_{c_{n}}$ is a ground state for $\mathscr{E}_{c_{n}}(\psi)$, we deduce

$$
\mathscr{E}_{c_{n}}\left(Q_{c_{n}}\right) \leq E_{\mathrm{nr}}(N)+N m c_{n}^{2},
$$

with $E_{\mathrm{nr}}(N)$ defined in (3-4), so that (3-7) gives us

$$
-\mu_{c_{n}} N \leq E_{\mathrm{nr}}(N)+N m c_{n}^{2} .
$$

From [Lieb 1977] we know that $E_{\mathrm{nr}}(N)<0$ and thus $\delta_{2}=-E_{\mathrm{nr}}(N) / N>0$ is a legitimate choice.

To prove the existence of $\delta_{1}>0$, we observe that each $Q_{c_{n}} \geq 0$ is the ground state of the "relativistic" Schrödinger operator

$$
H_{c_{n}}=\sqrt{-c_{n}^{2} \Delta+m^{2} c_{n}^{4}}-\left(|x|^{-1} *\left|Q_{c_{n}}\right|^{2}\right) .
$$

Since all $Q_{c_{n}}$ are radial functions with $\left\|Q_{c_{n}}\right\|_{L^{2}}^{2}=N$ for all $n \geq 1$, we can invoke Newton's theorem to find

$$
\int_{\mathbb{R}^{3}} \frac{\left|Q_{c_{n}}(y)\right|^{2}}{|x-y|} d y \leq \frac{N}{|x|} .
$$

By the min-max principle, we infer the lower bound

where

$$
-\mu_{c_{n}} \geq \inf \sigma\left(\bar{H}_{c_{n}}\right)
$$

$$
\bar{H}_{c_{n}}=\sqrt{-c_{n}^{2} \Delta+m^{2} c_{n}^{4}}-\frac{N}{|x|} \text {. }
$$


From [Herbst 1977] and reinstalling the speed of light $c>0$ there, we recall that we have inf $\sigma\left(\bar{H}_{c_{n}}\right)>$ $-\infty$ if and only if $N<(2 / \pi) c_{n}$. Thus $\bar{H}_{c_{n}}$ is bounded below for $n \gg 1$ and, moreover, we have an explicit lower bound (see [Herbst 1977] again) given by

$$
\inf \sigma\left(\bar{H}_{c_{n}}\right) \geq m c_{n}^{2} \sqrt{1-\left(\frac{\pi N}{2 c_{n}}\right)^{2}}
$$

Since $\sqrt{1-x^{2}} \geq 1-x^{2}$ for $|x| \leq 1$, we conclude

$$
-\mu_{c_{n}} \geq m c_{n}^{2}\left(1-\left(\frac{\pi N}{2 c_{n}}\right)^{2}\right)=m c_{n}^{2}-\frac{1}{4} m \pi^{2} N^{2}, \quad \text { for all } n \geq n_{0},
$$

provided that $n_{0} \gg 1$. By choosing $\delta_{1}=\frac{1}{4} m \pi^{2} N^{2}>0$, we complete the proof of Lemma 2 .

Next, we derive an a priori bound on the sequence of ground states.

Lemma 3. Let $\left\{Q_{c_{n}}\right\}_{n=1}^{\infty}$ be as in Proposition 1. Then there exists a constant $M>0$ such that

$$
\left\|Q_{c_{n}}\right\|_{H^{1}} \leq M, \quad \text { for all } n \geq 1 .
$$

Proof. Since $\left\|Q_{c_{n}}\right\|_{L^{2}}^{2}=N$ for all $n \geq 1$, we only have to derive a uniform bound for $\left\|\nabla Q_{c_{n}}\right\|_{L^{2}}$ which can be done as follows. From (3-6) we obtain

$$
\begin{aligned}
c_{n}^{2} \| \nabla & Q_{c_{n}}\left\|_{L^{2}}^{2}+m^{2} c_{n}^{4}\right\| Q_{c_{n}} \|_{L^{2}}^{2} \\
& =\left\langle\sqrt{-c_{n}^{2} \Delta+m^{2} c_{n}^{4}} Q_{c_{n}}, \sqrt{-c_{n}^{2} \Delta+m^{2} c_{n}^{4}} Q_{c_{n}}\right\rangle \\
& \leq \mu_{c_{n}}^{2}\left\langle Q_{c_{n}}, Q_{c_{n}}\right\rangle+2\left|\mu_{c_{n}}\right|\left\langle Q_{c_{n}},\left(|x|^{-1} *\left|Q_{c_{n}}\right|^{2}\right) Q_{c_{n}}\right\rangle+\left\langle Q_{c_{n}}\left(|x|^{-1} *\left|Q_{c_{n}}\right|^{2}\right),\left(|x|^{-1} *\left|Q_{c_{n}}\right|^{2}\right) Q_{c_{n}}\right\rangle .
\end{aligned}
$$

To bound the terms on the right, we notice that Kato's inequality $|x|^{-1} \lesssim|\nabla|$ implies

$$
\left\||x|^{-1} *\left|Q_{c_{n}}\right|^{2}\right\|_{L^{\infty}} \lesssim\left\langle Q_{c_{n}},|\nabla| Q_{c_{n}}\right\rangle \lesssim\left\|Q_{c_{n}}\right\|_{L^{2}}\left\|\nabla Q_{c_{n}}\right\|_{L^{2}}
$$

Using this bound, Hölder's inequality, and the bound $\left|\mu_{c_{n}}\right| \leq m c_{n}^{2}$ for $n \gg 1$ from Lemma 2, we obtain

$$
c_{n}^{2}\left\|\nabla Q_{c_{n}}\right\|_{L^{2}}^{2} \lesssim m c_{n}^{2} N^{3 / 2}\left\|\nabla Q_{c_{n}}\right\|_{L^{2}}+N^{2}\left\|\nabla Q_{c_{n}}\right\|_{L^{2}}^{2}
$$

for $n \gg 1$. Since $c_{n} \rightarrow \infty$ and $N$ is fixed, we conclude that there exists $M>0$ such that

$$
\left\|\nabla Q_{c_{n}}\right\|_{L^{2}} \leq M
$$

for $n \gg 1$. By choosing $M>0$ possibly larger, we extend this bound to all $n \geq 1$.

We now come the proof of Proposition 1 itself. By the a priori bound in Lemma 3, we have (after possibly passing to a subsequence) that

$$
Q_{c_{n}} \rightarrow Q_{\infty} \text { in } H^{1}\left(\mathbb{R}^{3}\right) \text { and } Q_{c_{n}}(x) \rightarrow Q_{\infty}(x) \text { for a. e. } x \in \mathbb{R}^{3} \text { as } n \rightarrow \infty,
$$

for some $Q_{\infty} \in H^{1}\left(\mathbb{R}^{3}\right)$. By radiality and strict positivity of all the $Q_{c_{n}}$, it follows that $Q_{\infty}(|x|) \geq 0$ is a radial and nonnegative function. Furthermore, since $\left\{Q_{c_{n}}\right\}_{n=1}^{\infty}$ forms a sequence of radial functions on $\mathbb{R}^{3}$ with a uniform $H^{1}$-bound, a classical result (see [Strauss 1977]) yields that

$$
Q_{c_{n}} \rightarrow Q_{\infty} \text { in } L^{p}\left(\mathbb{R}^{3}\right) \text { as } n \rightarrow \infty \text { for any } 2<p<6
$$


By Lemma 2, we have that $\left\{-\mu_{c_{n}}-m c_{n}^{2}\right\}_{n=1}^{\infty}$ is a bounded sequence, which is also uniformly bounded away from 0 . Hence extracting a suitable subsequence yields

$$
\lim _{n \rightarrow \infty}\left(-\mu_{c_{n}}-m c_{n}^{2}\right)=-\lambda<0
$$

for some $\lambda>0$.

Using that $Q_{c_{n}} \rightarrow Q_{\infty}$ in $H^{1}$ and the strong convergence (3-8), we can pass to the limit in (3-6) and find that the radial, nonnegative function $Q_{\infty} \in H^{1}\left(\mathbb{R}^{3}\right)$ satisfies

$$
-\frac{1}{2 m} \Delta Q_{\infty}-\left(|x|^{-1} *\left|Q_{\infty}\right|^{2}\right) Q_{\infty}=-\lambda Q_{\infty} \quad \text { in } H^{-1}\left(\mathbb{R}^{3}\right) .
$$

When taking this limit, we use the fact that

$$
\lim _{n \rightarrow \infty}\left\langle f,\left(\sqrt{-c_{n}^{2} \Delta+m^{2} c_{n}^{4}}-m c_{n}^{2}+\frac{1}{2 m} \Delta\right) Q_{c_{n}}\right\rangle=0 \quad \text { for all } f \in H^{1}\left(\mathbb{R}^{3}\right),
$$

which is easy to verify for test functions $f \in C_{0}^{\infty}\left(\mathbb{R}^{3}\right)$ by taking the Fourier transform and using that

$$
\sqrt{c_{n}^{2} \xi^{2}+m^{2} c_{n}^{4}}-m c_{n}^{2}-\frac{\xi^{2}}{2 m} \rightarrow 0 \text { for every } \xi \in \mathbb{R}^{3} \text { as } c_{n} \rightarrow \infty
$$

The claim above extends to all $f \in H^{1}\left(\mathbb{R}^{3}\right)$ by a simple density argument.

Next we prove that in fact $\int\left|Q_{\infty}\right|^{2}=N$ holds, which a-posteriori would show that $Q_{c_{n}} \rightarrow Q_{\infty}$ strongly in $L^{2}\left(\mathbb{R}^{3}\right)$. To prove this claim, we note that Equation (3-6) and its limit (3-10) give us

$$
\left(-\mu_{c_{n}}-m c_{n}^{2}\right) N=\frac{1}{2 m} \int_{\mathbb{R}^{3}}\left|\nabla Q_{c_{n}}\right|^{2}-\int_{\mathbb{R}^{3}}\left(|x|^{-1} *\left|Q_{c_{n}}\right|^{2}\right)\left|Q_{c_{n}}\right|^{2}+r_{n},
$$

with $r_{n} \rightarrow 0$ as $n \rightarrow \infty$. Note that the right-hand side is not weakly lower semicontinuous (with respect to weak $H^{1}$-convergence), unlike the case of atomic Hartree and Hartree-Fock energy functionals. To deal with the non weakly lower semicontinuous part given by the potential energy term, we use (3-8) again and the Hardy-Littlewood-Sobolev inequality. Then, by the weak lower semicontinuity of the kinetic energy term in (3-11), we deduce from (3-11) and (3-10) that

$$
-\lambda N \geq \frac{1}{2 m} \int_{\mathbb{R}^{3}}\left|\nabla Q_{\infty}\right|^{2}-\int_{\mathbb{R}^{3}}\left(|x|^{-1} *\left|Q_{\infty}\right|^{2}\right)\left|Q_{\infty}\right|^{2}=-\lambda \int_{\mathbb{R}^{3}}\left|Q_{\infty}\right|^{2} .
$$

Because of $\lambda>0$, we see that $\int\left|Q_{\infty}\right|^{2} \geq N$ must hold. On the other hand, we have $N \geq \int\left|Q_{\infty}\right|^{2}$ by the weak $L^{2}$-convergence. Thus we have $\int\left|Q_{\infty}\right|^{2}=N$ and, consequently,

$$
Q_{c_{n}} \rightarrow Q_{\infty} \text { in } L^{2}\left(\mathbb{R}^{3}\right) \text { as } n \rightarrow \infty .
$$

By Lemma 9 and a simple scaling argument, we see that $Q_{\infty}$ is the unique radial, nonnegative solution to (3-10) with $\int\left|Q_{\infty}\right|^{2}=N$. Here $\lambda>0$ is determined through $Q_{\infty}$, and $Q_{\infty}$ is in fact strictly positive.

It remains to show that

$$
Q_{c_{n}} \rightarrow Q_{\infty} \text { in } H^{1}\left(\mathbb{R}^{3}\right) \text { as } n \rightarrow \infty .
$$

To see this, we verify that $\left\{Q_{c_{n}}\right\}_{n=1}^{\infty}$ with $\int\left|Q_{c_{n}}\right|^{2}=N$ furnishes a minimizing sequence for the nonrelativistic Hartree energy $\mathscr{E}_{\mathrm{nr}}(\psi)$ subject to $\int|\psi|^{2}=N$, that is, for problem (3-4). Indeed, using (3-11) 
and (3-9) as well as the strong convergence (3-8) to pass to the limit in the potential energy, we deduce that

$$
\mathscr{E}_{\mathrm{nr}}\left(Q_{c_{n}}\right) \rightarrow-\lambda N+\frac{1}{2} \int_{\mathbb{R}^{3}}\left(|x|^{-1} *\left|Q_{\infty}\right|^{2}\right)\left|Q_{\infty}\right|^{2} \quad \text { as } \quad n \rightarrow \infty .
$$

However, this limit for $\mathscr{E}_{\mathrm{nr}}\left(Q_{c_{n}}\right)$ is equal to $\mathscr{E}_{\mathrm{nr}}\left(Q_{\infty}\right)$, as can be seen by multiplying (3-10) with $Q_{\infty}$ and integrating. Hence $\left\{Q_{c_{n}}\right\}_{n=1}^{\infty}$ is a minimizing sequence for problem (3-4). Next, we notice that standard concentration-compactness methods yield relative compactness in $H^{1}\left(\mathbb{R}^{3}\right)$ for any radial minimizing sequence for problem (3-4), which has a unique radial, nonnegative minimizer $Q_{\infty}$. Therefore (after possibly passing to another subsequence) we deduce that (3-13) holds.

To conclude the proof of Proposition 1, we note that we have convergence along every subsequence because of the uniqueness of the limit point $Q_{\infty} \in H^{1}\left(\mathbb{R}^{3}\right)$.

\section{Radial nondegeneracy of nonrelativistic ground states}

We consider the linear operator

$$
L_{+} \xi=-\frac{1}{2 m} \Delta \xi+\lambda \xi-\left(|x|^{-1} *\left|Q_{\infty}\right|^{2}\right) \xi-2 Q_{\infty}\left(|x|^{-1} *\left(Q_{\infty} \xi\right)\right),
$$

where $Q_{\infty} \in H^{1}\left(\mathbb{R}^{3}\right)$ is the radial, positive solution taken from Proposition 1. By standard arguments, it follows that $L_{+}$is a self-adjoint operator acting on $L^{2}\left(\mathbb{R}^{3}\right)$ with domain $H^{2}\left(\mathbb{R}^{3}\right)$. In this section, we study the restriction of $L_{+}$acting on $L_{\text {rad }}^{2}\left(\mathbb{R}^{3}\right)$ (that is, the radial $L^{2}$-functions on $\mathbb{R}^{3}$ ).

As a main result, we prove the so-called nondegeneracy of $L_{+}$on $L_{\text {rad }}^{2}\left(\mathbb{R}^{3}\right)$; that is, the triviality of its kernel.

Proposition 2. For the linear operator $L_{+}$be given by (4-1), we have

$$
\operatorname{ker} L_{+}=\{0\} \quad \text { when } L_{+} \text {is restricted to } L_{\mathrm{rad}}^{2}\left(\mathbb{R}^{3}\right) \text {. }
$$

Remark. (1) As shown in Section 7 below, we will see that the triviality of the kernel of $L_{+}$on $L_{\text {rad }}^{2}\left(\mathbb{R}^{3}\right)$ implies

$$
\operatorname{ker} L_{+}=\operatorname{span}\left\{\partial_{x_{1}} Q_{\infty}, \partial_{x_{2}} Q_{\infty}, \partial_{x_{3}} Q_{\infty}\right\}
$$

For linearized operators arising from ground states for NLS with local nonlinearities, this fact is wellknown; see [Chang et al. 2007; Weinstein 1985]. However, the proof given there cannot be adapted to $L_{+}$given by (4-1) due to its nonlocal component. We refer to Section 7 for further details.

(2) Numerical evidence indicating that 0 is not an eigenvalue of $L_{+}$when restricted to radial functions can be found in [Harrison et al. 2003].

4.1. Proof of Proposition 2. Suppose that $Q_{\infty} \in H^{1}\left(\mathbb{R}^{3}\right)$ is the unique radial, positive solution to (3-3) with $\int\left|Q_{\infty}\right|^{2}=N$ for some $N>0$ given. In what follows, it will be convenient and without loss of generality to assume that $Q_{\infty}$ satisfies

$$
-\Delta Q_{\infty}-\left(|x|^{-1} *\left|Q_{\infty}\right|^{2}\right) Q_{\infty}=-Q_{\infty}
$$


which amounts to rescaling $Q_{\infty}(x) \mapsto a Q_{\infty}(b x)$ with suitable $a>0$ and $b>0$. Likewise, the linear operator $L_{+}$then reads

$$
L_{+} \xi=-\Delta \xi+\xi-\left(|x|^{-1} *\left|Q_{\infty}\right|^{2}\right) \xi-2 Q_{\infty}\left(|x|^{-1} *\left(Q_{\infty} \xi\right)\right) .
$$

Recall that we restrict ourselves to radial $\xi \in L_{\text {rad }}^{2}\left(\mathbb{R}^{3}\right)$. Therefore, we can rewrite the nonlocal term in $L_{+}$by invoking Newton's theorem in $\mathbb{R}^{3}$ (see [Lieb and Loss 2001, Theorem 9.7]): For any radial function $\rho=\rho(|x|)$ such that $\rho \in L^{1}\left(\mathbb{R}^{3},(1+|x|)^{-1} d x\right)$, we have

$$
-\left(|x|^{-1} * \rho\right)(r)=\int_{0}^{r} K(r, s) \rho(s) d s-\int_{\mathbb{R}^{3}} \frac{\rho(|x|)}{|x|},
$$

for $r=|x| \geq 0$, where $K(r, s)$ is given by

$$
K(r, s)=4 \pi s\left(1-\frac{s}{r}\right) \geq 0, \quad \text { for } r \geq s .
$$

Since the ground state $Q_{\infty}$ is exponentially decaying, we can apply Newton's theorem to $\rho=Q_{\infty} \xi$ for any $\xi \in L_{\mathrm{rad}}^{2}\left(\mathbb{R}^{3}\right)$ and obtain the following result.

Lemma 4. For any $\xi \in L_{\mathrm{rad}}^{2}\left(\mathbb{R}^{3}\right)$, we have

$$
L_{+} \xi=\mathscr{L}_{+} \xi-2 Q_{\infty}\left(\int_{\mathbb{R}^{3}} \frac{Q_{\infty} \xi}{|x|}\right),
$$

where $\mathscr{L}_{+}$is given by

$$
\mathscr{L}_{+} \xi=-\Delta \xi+\xi-\left(|x|^{-1} *\left|Q_{\infty}\right|^{2}\right) \xi+W \xi,
$$

with

$$
(W \xi)(r)=2 Q_{\infty}(r) \int_{0}^{r} K(r, s) Q_{\infty}(s) \xi(s) d s .
$$

The following auxiliary result shows exponential growth of solutions $v$ to the linear equation $\mathscr{L}_{+} v=0$.

Lemma 5. Suppose the radial function $v=v(r)$ solves $\mathscr{L}_{+} v=0$ with $v(0) \neq 0$ and $v^{\prime}(0)=0$. Then the function $v(r)$ has no sign change and $v(r)$ grows exponentially as $r \rightarrow \infty$. More precisely, for any $0<\delta<1$, there exist constants $C>0$ and $R>0$ such that

$$
|v(r)| \geq C e^{+\delta r}, \quad \text { for all } r \geq R .
$$

In particular, we have that $v \notin L_{\mathrm{rad}}^{2}\left(\mathbb{R}^{3}\right)$.

Proof. Since $\mathscr{L}_{+} v=0$ is a linear equation, we can assume without loss of generality that $v(0)>0$; and moreover it is convenient to assume that $v(0)>Q_{\infty}(0)$ holds. Next, we write $\mathscr{L}_{+} v=0$ as

with

$$
v^{\prime \prime}(r)+\frac{2}{r} v^{\prime}(r)=V(r) v(r)+W(r),
$$

$$
\begin{aligned}
V(r) & =1-\left(|x|^{-1} *\left|Q_{\infty}\right|^{2}\right)(r), \\
W(r) & =2 Q_{\infty}(r) \int_{0}^{r} K(r, s) Q_{\infty}(s) v(s) d s .
\end{aligned}
$$


Note that $Q_{\infty}(r)$ satisfies (4-10) with $W(r)$ being removed, that is,

$$
Q_{\infty}^{\prime \prime}(r)+\frac{2}{r} Q_{\infty}^{\prime}(r)=V(r) Q_{\infty}(r)
$$

We now compare $v(r)$ and $Q_{\infty}(r)$ as follows. An elementary calculation, using equations (4-10) and (4-13), leads to the "Wronskian-type" identity

$$
\left(r^{2}\left(Q_{\infty} v^{\prime}-Q_{\infty}^{\prime} v\right)\right)^{\prime}=r^{2} Q_{\infty} W
$$

which, by integration, gives us

$$
r^{2}\left(Q_{\infty} v^{\prime}-Q_{\infty}^{\prime} v\right)(r)=\int_{0}^{r} s^{2} Q_{\infty}(s) W(s) d s .
$$

Hence, while keeping in mind that $Q_{\infty}(r)>0$, we find

$$
r^{2}\left(\frac{v(r)}{Q_{\infty}(r)}\right)^{\prime}=\frac{1}{Q_{\infty}(r)^{2}} \int_{0}^{r} s^{2} Q_{\infty}(s) W(s) d s .
$$

From this identity we now claim that

$$
v(r)>Q_{\infty}(r), \quad \text { for all } r \geq 0 .
$$

To see this, recall that $v(0)>Q_{\infty}(0)$ and, by continuity, we have that $v(r)>Q(r)$ for $r>0$ sufficiently small. Suppose now, on the contrary to (4-17), that there is a first intersection at some positive $r=r_{*}$, say, so that $v\left(r_{*}\right)=Q_{\infty}\left(r_{*}\right)$. It is easy to see that the left-hand side of (4-16) (or equivalently (4-15)) has to be $\leq 0$ at $r=r_{*}$. On the other hand, since $v(r)>Q_{\infty}(r)>0$ on $\left[0, r_{*}\right)$, we conclude that the integral on right-hand side of (4-16) at $r=r_{*}$ must be strictly positive. This contradiction shows that (4-17) must hold. In particular, the function $v(r)$ never changes its sign.

Next, we insert the estimate (4-17) back into (4-16), which yields

$$
r^{2}\left(\frac{v(r)}{Q(r)}\right)^{\prime}(r) \geq \frac{2}{Q_{\infty}(r)^{2}} \int_{0}^{r} s^{2} Q_{\infty}(s)^{2} \int_{0}^{s} K(s, t) Q_{\infty}(t)^{2} d t d s .
$$

We notice that $Q_{\infty}(r)>0$ is the unique ground state for the Schrödinger operator

$$
H=-\Delta+\widetilde{V}, \quad \text { with } \widetilde{V}=-|x|^{-1} *\left|Q_{\infty}\right|^{2} .
$$

Since $H Q_{\infty}=-Q_{\infty}$ and $\widetilde{V}$ is a continuous function with $\widetilde{V} \rightarrow 0$ as $|x| \rightarrow \infty$, standard arguments show that, for any $\varepsilon>0$, there exists a constant $A_{\varepsilon}>0$ such that

$$
Q_{\infty}(r) \leq A_{\varepsilon} e^{-(1-\varepsilon) r}, \quad \text { for all } r \geq 0 .
$$

Furthermore, since $Q_{\infty}(r)>0$ is the ground state of $H$, we can obtain the following lower bound: For any $\varepsilon>0$, there exists a constant $B_{\varepsilon}>0$ such that

$$
Q_{\infty}(r) \geq B_{\varepsilon} e^{-(1+\varepsilon) r}, \quad \text { for all } r \geq 0 .
$$

For this classical result on ground states for Schrödinger operators. See, for example, [Carmona and Simon 1981, Theorem 3.2] where a probabilistic proof is given. 
Now let $0<\varepsilon<1$ be given. Inserting the bounds (4-20) and (4-21) into Equation (4-18), we obtain

$$
r^{2}\left(\frac{v(r)}{Q(r)}\right)^{\prime}(r) \geq C e^{(2-2 \varepsilon) r} \int_{0}^{r} s^{2} e^{-(2+2 \varepsilon) s} \int_{0}^{s} K(s, t) e^{-(2+2 \varepsilon) t} d t d s,
$$

with some constant $C=C_{\varepsilon}>0$ (we drop its dependence on $\varepsilon$ henceforth). Since the double integral on the right-hand side converges as $r \rightarrow \infty$ to some finite positive value, there exists some $a>0$ such that

$$
r^{2}\left(\frac{v(r)}{Q(r)}\right)^{\prime}(r) \geq C e^{(2-2 \varepsilon) r}, \quad \text { for all } r \geq a,
$$

with some constant $C>0$. Integrating this lower bound and using (4-21) again, we find that

$$
v(r) \geq C \frac{e^{(1-3 \varepsilon) r}}{r^{2}}, \quad \text { for all } r \geq R,
$$

with some constants $C>0$ and $R \gg 1$. Thus, for any $0<\delta<1$, we arrive at the claim of Lemma 5 by taking $0<\varepsilon<\frac{1}{3}(1-\delta)$ and choosing $C>0$ appropriately.

With the help of Lemma 5 we are now able to prove the triviality of the kernel of $L_{+}$in the radial sector.

Lemma 6. For $L_{+}$be given by (4-1), we have that $L_{+} \xi=0$ with $\xi \in L_{\mathrm{rad}}^{2}\left(\mathbb{R}^{3}\right)$ implies that $\xi \equiv 0$.

Proof. Suppose there exists $\xi \in L_{\mathrm{rad}}^{2}\left(\mathbb{R}^{3}\right)$ with $\xi \not \equiv 0$ such that $L_{+} \xi=0$. Then, by Lemma 4 , the function $\xi$ solves the inhomogeneous problem

$$
\mathscr{L}_{+} \xi=2 \sigma Q_{\infty}, \quad \text { with } \sigma=\int_{\mathbb{R}^{3}} \frac{Q_{\infty} \xi}{|x|} .
$$

Therefore,

$$
\xi=v+w
$$

where $w$ is any particular solution to (4-25) and $v$ is some function such that $\mathscr{L}_{+} v=0$. As shown below, it suffices to restrict ourselves to smooth $v$ and $w$.

We shall now construct a smooth $w \in L_{\text {rad }}^{2}\left(\mathbb{R}^{3}\right)$ as follows. We define the smooth radial function

$$
R=2 Q_{\infty}+r \partial_{r} Q_{\infty} \in L_{\mathrm{rad}}^{2}\left(\mathbb{R}^{3}\right),
$$

where a calculation shows that

$$
L_{+} R=-2 Q_{\infty}
$$

Furthermore, by applying Lemma 4 to $R$, we find

$$
\mathscr{L}_{+} R=2(\tau-1) Q_{\infty}, \quad \text { with } \tau=\int_{\mathbb{R}^{3}} \frac{Q_{\infty} R}{|x|} .
$$

Note that $\tau \neq 1$ must hold, for otherwise Lemma 5 with $v=R$ (and $v(0)=R(0)=Q(0)>0$ and $v^{\prime}(0)=R^{\prime}(0)=0$ ) would yield that $R \notin L_{\mathrm{rad}}^{2}\left(\mathbb{R}^{3}\right)$, which is a contradiction. Thus we have found a smooth particular solution to (4-25) given by

$$
w=\frac{\sigma}{\tau-1} R \in L_{\mathrm{rad}}^{2}\left(\mathbb{R}^{3}\right)
$$


Further, we notice that $\xi \in L_{\mathrm{rad}}^{2}\left(\mathbb{R}^{3}\right)$ with $L_{+} \xi=0$ is smooth by bootstrapping this equation. Therefore, by (4-26), we conclude that $v$ has to be smooth as well. Suppose that $v \equiv 0$. Then we have $\xi=w$ and $\sigma \neq 0$ (since otherwise $w=0 \neq \xi$ ). This, however, contradicts that $L_{+} \xi=0$ and $L_{+} w=-2(\sigma /(\tau-1)) Q_{\infty} \neq 0$.

Thus we see that $v \not \equiv 0$ in (4-26), where $v^{\prime}(0)=0$ by smoothness of $v$. Suppose now that $v(0) \neq 0$. Then Lemma 5 yields that $v \notin L_{\text {rad }}^{2}\left(\mathbb{R}^{3}\right)$, which contradicts (4-26) together with the fact that $\xi$ and $w$ both belong to $L_{\text {rad }}^{2}\left(\mathbb{R}^{3}\right)$. Finally, suppose that $v(0)=0$ holds. Then $v$ solves the equation $\mathscr{L}_{+} v=0$ with initial data $v(0)=0$ and $v^{\prime}(0)=0$. However, by a standard fixed point argument, we see that the linear integro-differential equation $\mathscr{L}_{+} v=0$ with given initial data $v(0) \in \mathbb{R}$ and $v^{\prime}(0)=0$ has a unique solution. So $v(0)=0$ and $v^{\prime}(0)=0$ implies that $v \equiv 0$. Again, we arrive at a contradiction as above.

Clearly, Lemma 6 completes the proof of Proposition 2.

\section{Local uniqueness around $Q_{\infty}$}

Recall that $H_{\mathrm{r}}^{1}\left(\mathbb{R}^{3}\right)$ denotes space of radial and real-valued functions that belong to $H^{1}\left(\mathbb{R}^{3}\right)$. By using Proposition 2, we can now prove the following local uniqueness result for a small neighborhood around $Q_{\infty}$ in $H_{\mathrm{r}}^{1}\left(\mathbb{R}^{3}\right)$.

Proposition 3. Let $m>0$ and $N>0$ be given. Furthermore, suppose that $Q_{\infty} \in H_{\mathrm{r}}^{1}\left(\mathbb{R}^{3}\right)$ is the unique radial, positive solution to

$$
-\frac{1}{2 m} \Delta Q_{\infty}-\left(|x|^{-1} *\left|Q_{\infty}\right|^{2}\right) Q_{\infty}=-\lambda Q_{\infty}
$$

with $\int\left|Q_{\infty}\right|^{2}=N$, where $\lambda>0$ is determined through $Q_{\infty}$. Then there exist constants $c_{0} \gg 1, \varepsilon>0$, and $\delta>0$ such that the following holds. For any $(c, \mu)$ with

the equation

$$
c \geq c_{0}, \quad-\lambda-\varepsilon \leq-\mu-m c^{2} \leq-\lambda+\varepsilon,
$$

$$
\sqrt{-c^{2} \Delta+m^{2} c^{4}} Q-\left(|x|^{-1} *|Q|^{2}\right) Q=-\mu Q
$$

has a unique solution $Q \in H_{\mathrm{r}}^{1}\left(\mathbb{R}^{3}\right)$, provided that $\left\|Q-Q_{\infty}\right\|_{H^{1}} \leq \delta$.

5.1. Proof of Proposition 3. For $\beta \geq 0$ and $z>0$, we define the map

$$
G(u, \beta, z)=u+\mathscr{R}(\beta, z) g(u),
$$

where we set

$$
g(u)=-\left(|x|^{-1} *|u|^{2}\right) u,
$$

and, for $\beta \geq 0$ and $z>0$, we define the family of resolvents

$$
\mathscr{R}(\beta, z)= \begin{cases}(-(1 / 2 m) \Delta+z)^{-1} & \text { if } \beta=0, \\ \left(\sqrt{-\beta^{-2} \Delta+m^{2} \beta^{-4}}-m \beta^{-2}+z\right)^{-1} & \text { if } \beta>0 .\end{cases}
$$

By an elementary calculation, we verify the following equivalences:

$$
\begin{aligned}
& Q \in H_{\mathrm{r}}^{1}\left(\mathbb{R}^{3}\right) \text { solves }(5-1) \text { if and only if } G(Q, 0, \lambda)=0 ; \\
& Q \in H_{\mathrm{r}}^{1}\left(\mathbb{R}^{3}\right) \text { solves (5-2) if and only if } G\left(Q, c^{-1}, \mu+m c^{2}\right)=0 .
\end{aligned}
$$


To prove Proposition 3, we now construct an implicit function-type argument for the map

$$
G: H_{\mathrm{r}}^{1}\left(\mathbb{R}^{3}\right) \times\left[0, \beta_{0}\right] \times[\lambda-\varepsilon, \lambda+\varepsilon] \rightarrow H_{\mathrm{r}}^{1}\left(\mathbb{R}^{3}\right),
$$

where $\beta_{0}>0$ and $\varepsilon>0$ are small constants. To see that indeed $G(u, \beta, z) \in H_{\mathrm{r}}^{1}\left(\mathbb{R}^{3}\right)$ for $u \in H_{\mathrm{r}}^{1}\left(\mathbb{R}^{3}\right)$, we notice that $\mathscr{R}(\beta, z): H_{\mathrm{r}}^{1}\left(\mathbb{R}^{3}\right) \rightarrow H_{\mathrm{r}}^{1}\left(\mathbb{R}^{3}\right)$, as can be seen by using the Fourier transform. That $g(u)$ maps $H_{\mathrm{r}}^{1}\left(\mathbb{R}^{3}\right)$ into itself follows readily from the Hardy-Littlewood-Sobolev inequality and Sobolev embeddings. Hence (5-8) is indeed well-defined.

Next, we show that the derivative

$$
\partial_{u} G(u, \beta, z)=1+\mathscr{R}(\beta, z) \partial_{u} g(u): H_{\mathrm{r}}^{1}\left(\mathbb{R}^{3}\right) \rightarrow H_{\mathrm{r}}^{1}\left(\mathbb{R}^{3}\right)
$$

depends continuously on $(u, \beta, z)$. Here $\partial_{u} g(u)$ acting on $\xi \in H_{\mathrm{r}}^{1}\left(\mathbb{R}^{3}\right)$ is found to be

$$
\partial_{u} g(u) \xi=-\left(|x|^{-1} *|u|^{2}\right) \xi-2 u\left(|x|^{-1} *(u \xi)\right) .
$$

By using the Hardy-Littlewood-Sobolev inequality and Sobolev embeddings, we obtain that

$$
\left\|\left(\partial_{u} g\left(u_{1}\right)-\partial_{u} g\left(u_{2}\right)\right) \xi\right\|_{H^{1}} \lesssim\left(\left\|u_{1}\right\|_{H^{1}}+\left\|u_{2}\right\|_{H^{1}}\right)\left\|u_{1}-u_{2}\right\|_{H^{1}}\|\xi\|_{H^{1}}
$$

see, for example, [Lenzmann 2007] for similar estimates proving Lipschitz continuity of $g(u)$. Using this estimate, we find for $u_{1}, u_{2}, \xi \in H_{\mathrm{r}}^{1}\left(\mathbb{R}^{3}\right), \beta_{1}, \beta_{2} \in\left[0, \beta_{0}\right]$, and $z_{1}, z_{2}>0$,

$$
\begin{aligned}
\left\|\left(\partial_{u} G\left(u_{1}, \beta_{1}, z_{1}\right)-\partial_{u} G\left(u_{2}, \beta_{2}, z_{2}\right)\right) \xi\right\|_{H^{1}} \\
\leq\left\|\left(\Re\left(\beta_{1}, z_{1}\right)-\mathscr{R}\left(\beta_{2}, z_{2}\right)\right) \partial_{u} g\left(u_{1}\right) \xi\right\|_{H^{1}}+\left\|\mathscr{R}\left(\beta_{2}, z_{2}\right)\left(\partial_{u} g\left(u_{1}\right)-\partial_{u} g\left(u_{2}\right)\right) \xi\right\|_{H^{1}} \\
\quad \lesssim\left\|\Re\left(\beta_{1}, z_{1}\right)-\mathscr{R}\left(\beta_{2}, z_{2}\right)\right\|_{L^{2} \rightarrow L^{2}}\left\|u_{1}\right\|_{H^{1}}^{2}\|\xi\|_{H^{1}} \\
\quad+\left\|\Re\left(\beta_{2}, z_{2}\right)\right\|_{L^{2} \rightarrow L^{2}}\left(\left\|u_{1}\right\|_{H^{1}}+\left\|u_{2}\right\|_{H^{1}}\right)\left\|u_{1}-u_{2}\right\|_{H^{1}}\|\xi\|_{H^{1}}
\end{aligned}
$$

where we also use the fact that $\|\mathscr{R}(\beta, z)\|_{H^{s} \rightarrow H^{s}}=\|\mathscr{R}(\beta, z)\|_{L^{2} \rightarrow L^{2}}$ for any $s \in \mathbb{R}$, since $\mathscr{R}(\beta, z)$ commutes with $\langle\nabla\rangle$. Moreover, by using the Fourier transform, one verifies

$$
\left\|\mathscr{R}\left(\beta_{1}, z_{1}\right)-\mathscr{R}\left(\beta_{2}, z_{2}\right)\right\|_{L^{2} \rightarrow L^{2}} \rightarrow 0 \quad \text { as } \quad\left(\beta_{1}, z_{1}\right) \rightarrow\left(\beta_{2}, z_{2}\right),
$$

for any $\beta_{1}, \beta_{2} \geq 0$ and $z_{1}, z_{2}>0$. (For later use, we record that (5-13) also holds for complex $z_{1}, z_{2} \in$ $\mathbb{C} \backslash[0, \infty)$.) Going back to $(5-12)$, we thus find

$$
\left\|\partial_{u} G\left(u_{1}, \beta_{1}, z_{1}\right)-\partial_{u} G\left(u_{2}, \beta_{2}, z_{2}\right)\right\|_{H^{1} \rightarrow H^{1}} \rightarrow 0
$$

as $\left\|u_{1}-u_{2}\right\|_{H^{1}} \rightarrow 0$ and $\left(\beta_{1}, z_{1}\right) \rightarrow\left(\beta_{2}, z_{2}\right)$. Hence $\partial_{u} G(u, \beta, z)$ depends continuously on $(u, \beta, z)$.

By Proposition 2 and its following remark, we have that the radial restriction of the linearized operator $L_{+}$around $Q_{\infty}$ has trivial kernel. This implies that the compact operator $(-(1 /(2 m)) \Delta+\lambda)^{-1} \partial_{u} g\left(Q_{\infty}\right)$ does not have -1 in its spectrum. Hence the inverse operator

$$
\left(\partial_{u} G\left(Q_{\infty}, 0, \lambda\right)\right)^{-1}: H_{\mathrm{r}}^{1}\left(\mathbb{R}^{3}\right) \rightarrow H_{\mathrm{r}}^{1}\left(\mathbb{R}^{3}\right)
$$

exists. By the continuity of $\partial_{u} G(u, \beta, z)$ shown above, an appropriate version of an implicit function theorem (see, for example, [Chang 2005]) implies that, for $\beta_{0}>0$ and $\varepsilon>0$ sufficiently small, there 
exists a unique solution $Q=Q(\beta, z) \in H_{\mathrm{r}}^{1}\left(\mathbb{R}^{3}\right)$ such that

$$
G(Q(\beta, z), \beta, z)=0 \quad \text { for } \beta \in\left[0, \beta_{0}\right] \text { and } z \in[\lambda-\varepsilon, \lambda+\varepsilon]
$$

with

$$
\left\|Q(\beta, z)-Q_{\infty}\right\|_{H^{1}} \leq \delta \quad \text { for some } \delta>0 .
$$

Moreover, the map $(\beta, z) \mapsto Q(\beta, z) \in H_{\mathrm{r}}^{1}\left(\mathbb{R}^{3}\right)$ is continuous.

By setting $c_{0}=\beta_{0}^{-1}$ and recalling the equivalence (5-7), we complete the proof of Proposition 3.

\section{Proof of Theorem 2}

First, we notice that it is sufficient to prove uniqueness of symmetric-decreasing ground states for the variational problem (1-2), thanks to Theorem 1(iii). Next, we make use of the rescaling correspondence formulated in Lemma 1, which relates ground states for the dimensionalized and de-dimensionalized Hartree energy functionals $\mathscr{E}_{c}(\psi)$ and $\mathscr{E}(\psi)$ defined in (3-1) and (1-1), respectively.

In what follows, we fix $\int\left|Q_{c}\right|^{2}=1$ and we suppose that $Q_{c}=Q_{c}^{*} \in H^{1 / 2}\left(\mathbb{R}^{3}\right)$ is a symmetricdecreasing ground state for $\mathscr{E}_{c}(\psi)$ subject to $\int|\psi|^{2}=1$. Recall from Lemma 1 that $Q_{c}$ indeed exists for $c \geq c_{0}$ with $c_{0}$ being a sufficiently large constant. Let $\mu\left(Q_{c}\right)$ denote the Lagrange multiplier associated to $Q_{c}$ for $c \geq c_{0}$. We now claim that $\mu$ only depends on $c$ except for some countable set, that is, we have

$$
\mu\left(Q_{c}\right)=\mu(c), \quad \text { for } c \in\left(c_{0}, \infty\right) \backslash \Xi,
$$

where $\Xi$ is some countable set. To prove (6-1), we argue as follows. By Lemma 1, we see that $Q=$ $c^{-2} Q_{c}\left(c^{-1} \cdot\right)$ is a symmetric-decreasing ground state for $\mathscr{E}(\psi)$ subject to $\int|\psi|^{2}=N=c^{-1}$; and moreover the Lagrange multiplier $\mu(Q)$ for $Q$ is found to be

$$
\mu(Q)=c^{-2} \mu\left(Q_{c}\right) .
$$

Next, we consider the ground state energy $E(N)$ given by (1-2) for $0<N<c_{0}^{-1}$. From [Lieb and Yau 1987; Fröhlich et al. 2007b] we know that $E(N)$ is strictly concave. Hence $E^{\prime}(N)$ exists for all $N \in\left(0, c_{0}^{-1}\right) \backslash \Sigma$, where is $\Sigma$ is some countable set, and we readily find that

$$
E^{\prime}(N)=-\mu(Q), \quad \text { for } N \in\left(0, c_{0}^{-1}\right) \backslash \Sigma .
$$

Therefore the left-hand side of (6-2) only depends on $N=c^{-1}$ except when $N \in \Sigma$, which proves (6-1) with the countable set $\Xi=\left\{c: c>c_{0}\right.$ and $\left.c^{-1} \in \Sigma\right\}$.

Suppose $\left\{c_{n}\right\}_{n=1}^{\infty}$ is a sequence with such that $c_{n} \rightarrow \infty$ and values in $c_{n} \in\left(c_{0}, \infty\right) \backslash \Xi$. Correspondingly, let $\left\{Q_{c_{n}}\right\}_{n=1}^{\infty}$ be a sequence of symmetric-decreasing ground states for $\mathscr{E}_{c}(\psi)$ with $\int\left|Q_{c_{n}}\right|^{2}=1$ for all $n \geq 1$. By Proposition 1, for any such sequence $\left\{Q_{c_{n}}\right\}$, we have that $Q_{c_{n}}$ and its corresponding Lagrange multipliers $\mu_{c_{n}}$ satisfy the assumption of Proposition 3, provided that $n \gg 1$. By the local uniqueness result stated in Proposition 3 and the fact $\mu_{c_{n}}$ only depends on $c_{n}$, we conclude that the symmetricdecreasing ground state $Q_{c}$ for $\mathscr{E}_{c}(\psi)$ subject to $\int|\psi|^{2}=1$ is unique, provided that $c \in\left(c_{0}, \infty\right) \backslash \Xi$ holds, where $c_{0} \gg 1$ is sufficiently large and $\Xi$ is some countable set.

Finally, by Lemma 1, we deduce uniqueness of symmetric-decreasing ground states $Q$ for $\mathscr{E}(\psi)$ subject to $\int|\psi|^{2}=N$, provided that $N \in\left(0, N_{0}\right) \backslash \Sigma$ holds, where $N_{0}=c_{0}^{-1} \ll 1$ is sufficiently small and $\Sigma$ denotes some countable set. 


\section{Proof of Theorems 3 and 4}

We first prove Theorem 4. By rescaling $Q_{\infty}(r) \mapsto a Q_{\infty}(b r)$ with suitable $a>0$ and $b>0$, we can assume without loss of generality that $Q_{\infty} \in H_{\mathrm{r}}^{1}\left(\mathbb{R}^{3}\right)$ satisfies the normalized equation

$$
-\Delta Q_{\infty}-\left(|x|^{-1} *\left|Q_{\infty}\right|^{2}\right) Q_{\infty}=-Q_{\infty} .
$$

To complete the proof of Theorem 4 , it suffices to prove the following result.

Proposition 4. Let $Q_{\infty} \in H_{\mathrm{r}}^{1}\left(\mathbb{R}^{3}\right)$ be the unique radial and positive solution to Equation (7-1). Then the linearized operator $L_{+}$given by

$$
L_{+} \xi=-\Delta \xi+\xi-\left(|x|^{-1} *\left|Q_{\infty}\right|^{2}\right) \xi-2 Q_{\infty}\left(|x|^{-1} *\left(Q_{\infty} \xi\right)\right),
$$

acting on $L^{2}\left(\mathbb{R}^{3}\right)$ with domain $H^{1}\left(\mathbb{R}^{3}\right)$, has the kernel

$$
\operatorname{ker} L_{+}=\operatorname{span}\left\{\partial_{x_{1}} Q_{\infty}, \partial_{x_{2}} Q_{\infty}, \partial_{x_{3}} Q_{\infty}\right\} .
$$

Remark. For linearized operators $L_{+}$arising from ground states $Q$ for NLS with local nonlinearities, it is a well-known fact that $\operatorname{ker} L_{+}=\{0\}$ when $L_{+}$is restricted to radial functions implies that $\operatorname{ker} L_{+}$is spanned by $\left\{\partial_{x_{i}} Q\right\}_{i=1}^{3}$.

The proof, however, involves some Sturm-Liouville theory which is not applicable to $L_{+}$given above, due to the presence of the nonlocal term. (Also, recall that Newton's theorem is not at our disposal, since we do not restrict ourselves to radial functions anymore.) To overcome this difficulty, we have to develop Perron-Frobenius-type arguments for the action of $L_{+}$with respect to decomposition into spherical harmonics.

7.1. Proof of Proposition 4. Since $Q_{\infty}(r)$ and $|x|^{-1}$ are radial functions, the operator $L_{+}$commutes with rotations in $\mathbb{R}^{3}$; that is, we have that $\left(L_{+} \xi(R \cdot)\right)(x)=\left(L_{+} \xi\right)(R x)$ for all $R \in O(3)$. Therefore, we decompose any $\xi \in L^{2}\left(\mathbb{R}^{3}\right)$ using spherical harmonics according to

$$
\xi(x)=\sum_{\ell=0}^{\infty} \sum_{m=-\ell}^{\ell} f_{\ell m}(r) Y_{\ell m}(\Omega),
$$

where $x=r \Omega$ with $r=|x|$ and $\Omega \in \mathbb{S}^{2}$. This gives us the direct decomposition

$$
L^{2}\left(\mathbb{R}^{3}\right)=\bigoplus_{\ell=0}^{\infty} \mathscr{H}_{(\ell)},
$$

so that $L_{+}$acts invariantly on each

$$
\mathscr{H}_{(\ell)}=L^{2}\left(\mathbb{R}_{+}, r^{2} d r\right) \otimes \mathscr{Y}_{(\ell)} .
$$

Here $\mathscr{Y}_{(\ell)}=\operatorname{span}\left\{Y_{\ell m}\right\}_{m=-\ell}^{+\ell}$ denotes the $(2 \ell+1)$-dimensional eigenspace corresponding to the eigenvalue $\kappa_{\ell}=-\ell(\ell+1)$ of the spherical Laplacian $\Delta_{\mathbb{S}^{2}}$ acting on $L^{2}\left(\mathbb{S}^{2}\right)$.

Let us now find an explicit formula for the action of $L_{+}$on each $\mathscr{H}_{(\ell)}$. To this end, we recall the well-known the fact that

$$
-\Delta=-\partial_{r}^{2}-\frac{2}{r} \partial_{r}+\frac{\ell(\ell+1)}{r^{2}} \quad \text { on } \mathscr{H}_{(\ell)},
$$


as well as the multipole expansion

$$
\frac{1}{\left|x-x^{\prime}\right|}=4 \pi \sum_{\ell=0}^{\infty} \sum_{m=-\ell}^{+\ell} \frac{1}{2 \ell+1} \frac{r_{<}^{\ell}}{r_{>}^{\ell+1}} Y_{\ell m}(\Omega) Y_{\ell m}^{*}\left(\Omega^{\prime}\right)
$$

where $r_{<}=\min \left(|x|,\left|x^{\prime}\right|\right)$ and $r_{>}=\max \left(|x|,\left|x^{\prime}\right|\right)$. An elementary calculation leads to the following equivalence: We have that $L_{+} \xi=0$ if and only if

$$
L_{+,(\ell)} f_{\ell m}=0, \quad \text { for } \ell=0,1,2, \ldots \text { and } m=-\ell, \ldots,+\ell,
$$

with $\xi$ given by (7-2). Here the operator $L_{+,(\ell)}$ acting on $L^{2}\left(\mathbb{R}_{+}, r^{2} d r\right)$ is (formally) given by

$$
\left(L_{+,(\ell)} f\right)(r)=-f^{\prime \prime}(r)-\frac{2}{r} f^{\prime}(r)+\frac{\ell(\ell+1)}{r^{2}} f(r)+V(r) f(r)+\left(W_{(\ell)} f\right)(r),
$$

with the local potential

$$
V(r)=-\left(|x|^{-1} *\left|Q_{\infty}\right|^{2}\right)(r)
$$

and the nonlocal linear operator

$$
\left(W_{(\ell)} f\right)(r)=-\frac{8 \pi}{2 \ell+1} Q_{\infty}(r) \int_{0}^{\infty} \frac{r_{<}^{\ell}}{r_{>}^{\ell+1}} Q_{\infty}(s) f(s) s^{2} d s,
$$

where $r_{<}=\min (r, s)$ and $r_{>}=\max (r, s)$.

To prove Proposition 4 , it suffices to assume henceforth that $\ell \geq 1$ holds, since $L_{+,(0)} f=0$ implies that $f \equiv 0$ holds, by Proposition 2 above. Hence any nontrivial elements in the kernel of $L_{+}$can only belong to $\mathcal{H}_{(\ell)}$ with $\ell \geq 1$. Before we proceed, we show that each $L_{+,(\ell)}$ enjoys a Perron-Frobenius property as follows.

Lemma 7. For each $\ell \geq 1$, the operator $L_{+,(\ell)}$ is essentially self-adjoint on $C_{0}^{\infty}\left(\mathbb{R}_{+}\right) \subset L^{2}\left(\mathbb{R}_{+}, r^{2} d r\right)$ and bounded below. Moreover, each $L_{+,(\ell)}$ has the Perron-Frobenius property. That is, if $e_{0,(\ell)}$ denotes the lowest eigenvalue of $L_{+,(\ell)}$, then $e_{0,(\ell)}$ is simple and the corresponding eigenfunction $\phi_{0,(\ell)}(r)>0$ is strictly positive.

Remarks. (1) We have indeed the lower bound $L_{+,(\ell)} \geq 0$ for all $\ell \geq 1$. This follows from $\mathscr{H}_{(\ell)} \perp Q_{\infty}$ for $\ell \geq 1$ and the fact that $\left.L_{+}\right|_{Q_{\infty}^{\perp}} \geq 0$, which can be proven in the same way as for ground states for local NLS; see, for example, [Chang et al. 2007; Weinstein 1985].

(2) It is easy to see that $L_{+,(\ell)}$ has in fact infinitely many eigenvalues between 0 and 1 . Indeed, the lower bound $Q_{\infty}(r) \geq B_{\varepsilon} e^{-(1+\varepsilon) r}$ (see the proof of Lemma 5) leads, by using Newton's theorem, to the upper bound $V(r) \leq-\alpha r^{-1}$ with some $\alpha>0$. Furthermore, one finds that $\left\langle f, W^{(\ell)} f\right\rangle<0$ for $f \not \equiv 0$. Hence, we conclude

$$
L_{+,(\ell)} \leq-\partial_{r}^{2}-\frac{2}{r} \partial_{r}+1+\frac{\ell(\ell+1)}{r^{2}}-\frac{\alpha}{r}
$$

on $L^{2}\left(\mathbb{R}_{+}, r^{2} d r\right)$. From the well-known spectral properties of the hydrogen atom Hamiltonian, we infer that the operator on the right has infinitely many eigenvalues below 1 , and so does $L_{+,(\ell)}$ by the min-max principle. 
Proof of Lemma 7. Since $Q_{\infty}(r)$ is exponentially decaying, it is straightforward to verify that $W_{(\ell)}$ is a bounded operator. Also, we have that $V \in L^{\infty}$ holds. Thus $L_{+,(\ell)}$ is bounded below (see also the remark following Lemma 7). Furthermore, it is well-known that

$$
-\Delta_{(\ell)}=-\partial_{r}^{2}-\frac{2}{r} \partial_{r}+\frac{\ell(\ell+1)}{r^{2}}
$$

is essentially self-adjoint on $C_{0}^{\infty}\left(\mathbb{R}_{+}\right)$provided that $\ell \geq 1$. In fact, this follows from [Reed and Simon 1980, Theorem X.10 and Example 4] which shows that $-\partial_{r}^{2}-(2 / r) \partial_{r}+\ell(\ell+1) / r^{2}$ is essentially selfadjoint on $C_{0}^{\infty}\left(\mathbb{R}_{+}\right)$if $\ell(\ell+1) / r^{2} \geq 3 / 4 r^{2}$. Furthermore, by the Kato-Rellich theorem and the fact that $V$ and $W_{(\ell)}$ are bounded and self-adjoint, we deduce that $L_{+,(\ell)}=-\Delta_{(\ell)}+V+W_{(\ell)}$ is essentially self-adjoint on $C_{0}^{\infty}\left(\mathbb{R}_{+}\right)$as well.

The Perron-Frobenius property of $L_{+,(\ell)}$ can be shown as follows. First, we consider the kinetic energy part in $L_{+,(\ell)}$, where we find that

$$
e^{t \Delta_{(\ell)}} \text { is positivity improving on } L^{2}\left(\mathbb{R}_{+}, r^{2} d r\right) \text { for all } t>0 .
$$

(Recall that, by definition, this means that $e^{t \Delta_{(\ell)}} f>0$ when $f \geq 0$ with $f \not \equiv 0$.) Indeed, an argument given in Appendix B shows that the integral kernel of $e^{t \Delta_{(\ell)}}$ is strictly positive:

$$
e^{t \Delta(\ell)}(r, s)=\frac{1}{2 t} \sqrt{\frac{1}{r s}} e^{-\frac{r^{2}+s^{2}}{4 t}} I_{\ell+1 / 2}\left(\frac{r s}{2 t}\right)>0, \quad \text { for } r, s>0 .
$$

Here $I_{k}(z)$ denotes the modified Bessel function of the first kind of order $k$. For later use, we record that (7-12) and the formula (by functional calculus)

$$
\left(-\Delta_{(\ell)}+\mu\right)^{-1}=\int_{0}^{\infty} e^{-t \mu} e^{t \Delta_{(\ell)}} d t, \quad \text { for } \mu>0,
$$

immediately show that

$$
\left(-\Delta_{(\ell)}+\mu\right)^{-1} \text { is positivity improving on } L^{2}\left(\mathbb{R}_{+}, r^{2} d r\right) \text { for all } \mu>0 .
$$

Next, let $A_{(\ell)}$ denote the bounded self-adjoint operator

$$
A_{(\ell)}=V+W_{(\ell)},
$$

where $V$ and $W_{(\ell)}$ are defined in (7-9) and (7-10), respectively. Note that $A_{(\ell)}$ is nonlocal. Using that $Q_{\infty}(r)$ is strictly positive, we readily find that

$$
-A_{(\ell)} \text { is positivity improving on } L^{2}\left(\mathbb{R}_{+}, r^{2} d r\right) .
$$

This leads to the following auxiliary result.

Lemma 8. For $\mu \gg 1$, the resolvent

$$
\left(L_{+,(\ell)}+\mu\right)^{-1}=\left(-\Delta_{(\ell)}+A_{(\ell)}+\mu\right)^{-1}
$$

is positivity improving on $L^{2}\left(\mathbb{R}_{+}, r^{2} d r\right)$. 
Proof. For $\mu \gg 1$, we have

$$
\frac{1}{L_{+,(\ell)}+\mu}=\frac{1}{-\Delta_{(\ell)}+\mu} \frac{1}{1+A_{(\ell)}\left(-\Delta_{(\ell)}+\mu\right)^{-1}} .
$$

Since $A_{(\ell)}$ is bounded, we conclude that $\left\|A_{(\ell)}\left(-\Delta_{(\ell)}+\mu\right)^{-1}\right\|_{L^{2} \rightarrow L^{2}}<1$ for $\mu \gg 1$. Thus a Neumann expansion yields

$$
\frac{1}{L_{+,(\ell)}+\mu}=\frac{1}{-\Delta_{(\ell)}+\mu} \sum_{\nu=0}^{\infty}\left(-A_{(\ell)}\left(-\Delta_{(\ell)}+\mu\right)^{-1}\right)^{\nu},
$$

provided that $\mu \gg 1$. Next, we recall from (7-15) that $\left(-\Delta_{(\ell)}+\mu\right)^{-1}$ is positivity improving. By this fact and (7-17), we deduce from (7-18) that $\left(L_{+,(\ell)}+\mu\right)^{-1}$ must be positivity improving for $\mu \gg 1$. This completes the proof of Lemma 8.

We now return to the proof of Lemma 7, which we complete as follows. Let $\ell \geq 1$ be fixed and suppose $e_{0,(\ell)}=\inf \sigma\left(L_{+,(\ell)}\right)$ is the lowest eigenvalue. Furthermore, we choose $\mu \gg 1$ such that, by Lemma 8 ,

$$
B=\left(L_{+,(\ell)}+\mu\right)^{-1}
$$

is positivity improving on $L^{2}\left(\mathbb{R}_{+}, r^{2} d r\right)$. Clearly, the operator $B$ is bounded and self-adjoint, and its largest eigenvalue $\lambda_{0}=\sup \sigma(B)$ is given by $\lambda_{0}=\left(e_{(\ell), 0}+\mu\right)^{-1}$. Also, the corresponding eigenspaces of $L_{+,(\ell)}$ and $B$ coincide. Since $B$ is positivity improving (and hence ergodic), we can invoke [Reed and Simon 1978, Theorem XIII.43] to conclude that $\lambda_{0}$ is simple and that the corresponding eigenfunction $\phi_{(\ell), 0}(r)$ is strictly positive on $\mathbb{R}_{+}$. This proof of Lemma 7 is therefore complete.

Let us now come back to the proof of Proposition 4, stating that ker $L_{+}$is spanned by $\left\{\partial_{x_{i}} Q_{\infty}\right\}_{i=1}^{3}$. By differentiating the nonlinear equation satisfied by $Q_{\infty}$, we readily obtain that $L_{+} \partial_{x_{i}} Q_{\infty}=0$ for $i=1,2,3$. Since $\partial_{x_{i}} Q_{\infty}(r)=Q_{\infty}^{\prime}(r)\left(x_{i} / r\right) \in \mathcal{H}_{(1)}$, this show that

$$
L_{+,(1)} Q_{\infty}^{\prime}=0 \text {. }
$$

Furthermore, by monotonicity of $Q_{\infty}(r)$, we have that $Q_{\infty}^{\prime}(r) \leq 0$. Since $L_{+,(1)}$ is self-adjoint and $Q_{\infty}^{\prime}$ is an eigenfunction that does not change its sign, Lemma 7 shows that in fact $Q_{\infty}^{\prime}(r)=-\phi_{0,(1)}(r)$ holds, where $\phi_{0,(1)}>0$ is the strictly positive ground state of $L_{+,(1)}$, with $e_{0,(1)}=0$ being its corresponding eigenvalue. Therefore any $\xi \in \mathscr{H}_{(1)}$ such that $L_{+} \xi=0$ must be some linear combination of $\left\{\partial_{x_{i}} Q_{\infty}\right\}_{i=1}^{3}$.

To complete the proof of Proposition 4, we now claim that

$$
L_{+,(\ell)}>0, \quad \text { for } \ell \geq 2,
$$

which in particular shows that $L_{+} \xi=0$ with $\xi \in \mathscr{H}_{(\ell)}$ for some $\ell \geq 2$ implies that $\xi \equiv 0$. To prove (7-21), let $\ell \geq 2$ be fixed and set

$$
e_{0,(\ell)}=\inf \sigma\left(L_{+,(\ell)}\right) .
$$

Indeed, by the remark following Lemma 7, we know that $e_{0,(\ell)}<1$ is attained. (If $e_{0,(\ell)}$ was not attained, then $e_{0,(\ell)}=\inf \sigma_{\mathrm{ess}}\left(L_{+,(\ell)}\right)=1$ and (7-21) follows immediately.) By Lemma 7, the eigenvalue $e_{0,(\ell)}$ is simple and its corresponding eigenfunction $\phi_{0,(\ell)}(r)>0$ is strictly positive. Next, we notice that

$$
e_{0}=\left\langle\phi_{0,(\ell)}, L_{+,(\ell)} \phi_{0,(\ell)}\right\rangle=\left\langle\phi_{0,(\ell)}, L_{+,(1)} \phi_{0,(\ell)}\right\rangle+K_{(\ell)},
$$


where

$$
\begin{aligned}
K_{(\ell)}=\int_{0}^{\infty} \frac{(\ell(\ell+1)-2)}{r^{2}} \phi_{0,(\ell)}(r)^{2} r^{2} d r \\
\quad+8 \pi \int_{0}^{\infty} \int_{0}^{\infty} Q_{\infty}(r) \phi_{0,(\ell)}(r)\left(\frac{1}{3} \frac{r_{<}}{r_{>}^{2}}-\frac{1}{2 \ell+1} \frac{r_{<}^{\ell}}{r_{>}^{\ell+1}}\right) Q_{\infty}(s) \phi_{0,(\ell)}(s) r^{2} s^{2} d r d s,
\end{aligned}
$$

with $r_{<}=\min (r, s)$ and $r_{>}=\max (r, s)$. Using the strict positivity of $Q_{\infty}(r)$ and $\phi_{0,(\ell)}(r)$, we see that $K_{(\ell)}>0$ holds because of $\ell \geq 2$ and $\left(r_{<} / r_{>}\right) \leq 1$. Moreover, we recall from the preceding discussion that $L_{+,(1)} \geq e_{0,(1)}=0$. Therefore, by (7-23),

$$
e_{0,(\ell)} \geq K_{(\ell)}>0, \quad \text { for all } \ell \geq 2,
$$

which proves (7-21), completing the proof of Proposition 4, whence the proof of Theorem 4 follows.

7.2. Proof of Theorem 3. As in the proof of Theorem 2 above, it is convenient to fix $N>0$ and to consider symmetric-decreasing ground state $Q_{c} \in H_{\mathrm{r}}^{1}\left(\mathbb{R}^{3}\right)$ minimizing $\mathscr{E}_{c}(\psi)$ with $\int\left|Q_{c}\right|^{2}=N$, where we take $c>0$ sufficiently large. In what follows, let $\mu_{c}$ denote the Lagrange multiplier associated to $Q_{c}$. (It is possible that $\mu_{c}$ depends on $Q_{c}$ and not just on $c$.)

Recall from Proposition 1 that

$$
\left\|Q_{c}-Q_{\infty}\right\|_{H^{1}} \leq \delta_{1} \quad \text { and } \quad\left|-\mu_{c}-m c^{2}+\lambda\right| \leq \delta_{2},
$$

where $\delta_{1} \rightarrow 0$ and $\delta_{2} \rightarrow 0$ as $c \rightarrow \infty$. Here $Q_{\infty} \in H_{\mathrm{r}}^{1}\left(\mathbb{R}^{3}\right)$ is the unique radial positive solution to (3-3) with $\int\left|Q_{\infty}\right|^{2}=N$, where $\lambda>0$ is determined through $Q_{\infty}$. By Theorem 4 , the linear operator $L_{+}$given by

$$
L_{+} \xi=-\frac{1}{2 m} \Delta+\lambda-\left(|x|^{-1} *\left|Q_{\infty}\right|^{2}\right) \xi-2 Q_{\infty}\left(|x|^{-1} *\left(Q_{\infty} \xi\right)\right)
$$

has the kernel

$$
\operatorname{ker} L_{+}=\operatorname{span}\left\{\partial_{x_{1}} Q_{\infty}, \partial_{x_{2}} Q_{\infty}, \partial_{x_{3}} Q_{\infty}\right\} .
$$

Next, let $L_{+, c}$ denote the linear operators defined as

$$
L_{+, c} \xi=\sqrt{-c^{2} \Delta+m^{2} c^{4}} \xi+\mu_{c} \xi-\left(|x|^{-1} *\left|Q_{c}\right|^{2}\right) \xi-2 Q_{c}\left(|x|^{-1} *\left(Q_{c} \xi\right)\right) .
$$

Again, upon differentiating the Euler-Lagrange equation satisfied by $Q_{c}$, we see that $L_{+, c} \partial_{x_{i}} Q_{c}=0$ for $i=1,2,3$. Hence

$$
\operatorname{span}\left\{\partial_{x_{1}} Q_{c}, \partial_{x_{2}} Q_{c}, \partial_{x_{3}} Q_{c}\right\} \subseteq \operatorname{ker} L_{+, c} .
$$

By the following perturbation argument, we show that in fact equality holds for $c \gg 1$. By standard arguments, we see that $0 \in \sigma\left(L_{+}\right)$is an isolated eigenvalue. Thus we can construct the Riesz projection $P_{0}$ onto ker $L_{+}$by

$$
P_{0}=\frac{1}{2 \pi i} \oint_{\Gamma_{r}}\left(L_{+}-z\right)^{-1} d z,
$$

where the curve $\Gamma_{r}$ parametrizes the circle $\{z \in \mathbb{C}:|z|=r\}$. Here $r>0$ is chosen sufficiently small such that 0 is the only eigenvalue of $L_{+}$inside $|z| \leq r$. Next, we claim that the projection

$$
P_{0, c}=\frac{1}{2 \pi i} \oint_{\Gamma_{r}}\left(L_{+, c}-z\right)^{-1} d z
$$


exists for $c \gg 1$ and satisfies

$$
\left\|P_{0, c}-P_{0}\right\|_{L^{2} \rightarrow L^{2}} \rightarrow 0 \quad \text { as } \quad c \rightarrow \infty .
$$

Indeed, by using (7-25) and similar arguments as in the proof of Proposition 3 (see, for example, the resolvent estimate (5-13)), we conclude that

$$
\left\|\left(L_{+, c}-z\right)^{-1}\right\|_{L^{2} \rightarrow L^{2}} \leq C\left\|\left(L_{+}-z\right)^{-1}\right\|_{L^{2} \rightarrow L^{2}},
$$

for all $c \gg 1$ and $z \in \Gamma_{r}$, where $C>0$ is some constant. Furthermore, we have

$$
\left\|\left(L_{+, c}-z\right)^{-1}-\left(L_{+}-z\right)^{-1}\right\|_{L^{2} \rightarrow L^{2}} \rightarrow 0 \quad \text { as } \quad c \rightarrow \infty,
$$

for all $z \in \Gamma_{r}$. This shows that $P_{0, c}$ exists for $c \gg 1$ and that (7-32) holds. Since rank $P_{0}=3$ and the rank of $P_{0, c}$ remains constant for $c \gg 1$, by (7-32), we infer that $P_{0, c}$ has at most 3 eigenvalues (counted with their multiplicity) inside $|z| \leq r$, provided that $c \gg 1$. In particular, we conclude that $\operatorname{dim} \operatorname{ker} L_{+, c} \leq 3$ for $c \gg 1$. Therefore equality must hold in (7-29) whenever $c \gg 1$.

Thus we have found that $L_{+, c}$ has the desired kernel property if $c \gg 1$. By a rescaling argument formulated in Lemma 1, we conclude the analogous statement for the linear operator $L_{+}$arising from the unique symmetric-decreasing ground state $Q$ minimizing $\mathscr{E}(\psi)$ subject to $\int|\psi|^{2}=N$ with $N \ll 1$. The proof of Theorem 3 is now complete.

\section{Appendix A. Uniqueness of $Q_{\infty}$}

Suppose that $Q_{\infty} \in H^{1}\left(\mathbb{R}^{3}\right)$ solves

$$
-\frac{1}{2 m} \Delta Q_{\infty}-\left(|x|^{-1} *\left|Q_{\infty}\right|^{2}\right) Q_{\infty}=-\lambda Q_{\infty}
$$

with $m>0$ and $\lambda>0$ given. By rescaling $Q_{\infty}(r) \mapsto a Q_{\infty}(b r)$ with suitable $a>0$ and $b>0$, we can consider without loss of generality solutions $Q_{\infty} \in H^{1}\left(\mathbb{R}^{3}\right)$ to the "normalized" equation

$$
-\Delta Q_{\infty}-\left(|x|^{-1} *\left|Q_{\infty}\right|^{2}\right) Q_{\infty}=-Q_{\infty} .
$$

The following result is due to [Lieb 1977]; see also [Tod and Moroz 1999]. Here we provide a partly different proof, which is directly based on a comparison argument.

Lemma 9. Equation (A-2) has a unique radial, nonnegative solution $Q \in H_{\mathrm{r}}^{1}\left(\mathbb{R}^{3}\right)$ with $Q \not \equiv 0$. Moreover, we have that $Q(r)$ is in fact strictly positive.

Proof. Existence of a nonnegative, nontrivial solution $Q_{\infty} \in H_{\mathrm{r}}^{1}\left(\mathbb{R}^{3}\right)$ of (A-2) follows from variational arguments; see [Lieb 1977].

To prove that any nonnegative $Q \in H^{1}\left(\mathbb{R}^{3}\right)$, with $Q \not \equiv 0$, solving (A-2) is strictly positive, we can simply argue as follows. We rewrite (A-2) as

$$
Q(x)=\left((-\Delta+1)^{-1}(V Q)\right)(x)=\frac{1}{4 \pi} \int_{\mathbb{R}^{3}} \frac{e^{-|x-y|}}{|x-y|} V(y) Q(y) d y
$$

with $V=|x|^{-1} *|Q|^{2}$. Since $V \geq 0$ and $Q \geq 0$ (with $V \not \equiv 0$ and $Q \not \equiv 0$ ), Equation (A-3) shows that $Q$ is strictly positive. 
Let us now prove the claimed uniqueness. Suppose $Q \in H_{\mathrm{r}}^{1}\left(\mathbb{R}^{3}\right)$, with $Q \not \equiv 0$, is a solution to (A-2). Using Newton's theorem, we find that $Q(r)$ solves (after a suitable rescaling $Q(r) \mapsto a^{2} Q(a r)$ for some $a>0$; see [Lieb 1977]) the initial-value problem

$$
-v^{\prime \prime}(r)-\frac{2}{r} v^{\prime}(r)-v(r)+\left(\int_{0}^{r} K(r, s) v(s)^{2} d s\right) v(r)=0, \quad v(0)=v_{0}, \quad v^{\prime}(0)=0,
$$

with $v_{0}=Q(0) \in \mathbb{R}$. (Recall that $K(r, s) \geq 0$ is given by (4-6) above.) By standard fixed point arguments, we deduce that (A-4) has a unique local $C^{2}$-solution for given initial data $v(0) \in \mathbb{R}$ and $v^{\prime}(0)=0$, and $v(r)$ exists up to some maximal radius $R \in(0, \infty]$.

Suppose now that $Q \in H_{\mathrm{r}}^{1}\left(\mathbb{R}^{3}\right)$ and $\widetilde{Q} \in H_{\mathrm{r}}^{1}\left(\mathbb{R}^{3}\right)$ are two radial, nonnegative (and nontrivial) solutions to (A-2) with $Q \not \equiv \widetilde{Q}$. From the preceding discussion we know that $Q$ and $\widetilde{Q}$ are in fact strictly positive, and (after appropriate rescaling) both satisfy (A-4) with $v_{0}=Q(0)>0$ and $v_{0}=\widetilde{Q}(0)>0$, respectively. By uniqueness for (A-4), we conclude that $Q(0) \neq \widetilde{Q}(0)$ holds, since otherwise $Q \equiv \widetilde{Q}$. Therefore, we can henceforth assume that

$$
\widetilde{Q}(0)>Q(0) \text {. }
$$

Next, we notice that a calculation (similar to the one in the proof of Lemma 5) yields the integrated "Wronskian-type" identity

$$
r^{2}\left(Q(r) \widetilde{Q}^{\prime}(r)-Q^{\prime}(r) \widetilde{Q}(r)\right)=\int_{0}^{r} s^{2} Q(s) \widetilde{Q}(s)(\widetilde{V}(s)-V(s)) d s .
$$

Here,

$$
V(r)=\int_{0}^{r} K(r, s) Q(s)^{2} d s \text { and } \widetilde{V}(r)=\int_{0}^{r} K(r, s) \widetilde{Q}(s)^{2} d s .
$$

By continuity and (A-5), we have $\widetilde{Q}(r)>Q(r)$ at least initially for $r \geq 0$. Next, we conclude, by (A-6), that in fact

$$
\widetilde{Q}(r)>Q(r), \quad \text { for all } r \geq 0 .
$$

To see this, suppose on the contrary that $\widetilde{Q}(r)>0$ intersects $Q(r)>0$ for the first time at $r=r_{*}>0$, say. Then the left-hand side of (A-6) is found to be nonnegative at $r=r_{*}$, whereas the right-hand side must be strictly positive at $r=r_{*}$ since $\widetilde{V}(r)>V(r)$ on $\left(0, r_{*}\right)$. This contradiction shows that (A-8) holds.

Finally, we show that (A-8) leads to a contradiction (along the lines of [Lieb 1977]) as follows. To this end, we consider the Schrödinger operators

$$
H=-\Delta+V \quad \text { and } \quad \widetilde{H}=-\Delta+\widetilde{V},
$$

so that $H Q=Q$ and $\widetilde{H} \widetilde{Q}=\widetilde{Q}$. By standard theory of Schrödinger operators, we conclude that $Q$ and $\widetilde{Q}$ are (up to a normalization factor) the unique positive ground states (with eigenvalue $e=1$ ) for $H$ and $\widetilde{H}$, respectively. Therefore,

$$
\langle\phi, H \phi\rangle \geq\|\phi\|_{L^{2}}^{2} \quad \text { and } \quad\langle\phi, \widetilde{H} \phi\rangle \geq\|\phi\|_{L^{2}}^{2}, \quad \text { for } \phi \in H^{1}\left(\mathbb{R}^{3}\right),
$$

where equality holds if and only if $\phi=\lambda Q$ or $\phi=\lambda \widetilde{Q}$ for some $\lambda \in \mathbb{C}$, respectively.

Going back to (A-8), we find that $\widetilde{V}(r)>V(r)$ for all $r>0$, which leads to

$$
\|\widetilde{Q}\|_{L^{2}}^{2} \leq\langle\widetilde{Q}, H \widetilde{Q}\rangle=\langle\widetilde{Q}, \widetilde{H} \widetilde{Q}\rangle-\langle\widetilde{Q},(\widetilde{V}-V) \widetilde{Q}\rangle=\|\widetilde{Q}\|_{L^{2}}^{2}-\delta,
$$


for some $\delta>0$, which is a contradiction.

Hence (A-2) does not admit two different radial and nonnegative (and nontrivial) solutions $Q \in H_{\mathrm{r}}^{1}\left(\mathbb{R}^{3}\right)$ and $\widetilde{Q} \in H_{\mathrm{r}}^{1}\left(\mathbb{R}^{3}\right)$.

\section{Appendix B. Decomposition of $e^{t \Delta}$ using spherical harmonics}

Recall the explicit formula for the heat kernel of the Laplacian $\Delta$ on $\mathbb{R}^{3}$ :

$$
e^{t \Delta}(x, y)=\frac{1}{(4 \pi t)^{3 / 2}} e^{-|x-y|^{2} /(4 t)}=\frac{1}{(4 \pi t)^{3 / 2}} e^{-\left(x^{2}+y^{2}\right) /(4 t)} e^{(x \cdot y) /(2 t)} .
$$

Moreover, we have the well-known identity

$$
e^{a x \cdot y}=4 \pi \sum_{\ell=0}^{\infty} \sum_{m=-\ell}^{+\ell} i_{\ell}(a|x||y|) Y_{\ell m}(\Omega) Y_{\ell m}^{*}\left(\Omega^{\prime}\right)
$$

for $a>0, x=|x| \Omega$ and $y=|y| \Omega^{\prime}$ where $\Omega, \Omega^{\prime} \in \mathbb{S}^{2}$. Here

$$
i_{\ell}(z)=\sqrt{\frac{\pi}{2 z}} I_{\ell+1 / 2}(z)
$$

is the modified spherical Bessel function of the first kind of order $\ell$; whereas $I_{k}(z)$ denotes the modified Bessel function of the first kind of order $k$.

Let $\Delta_{(\ell)}$ denote the restriction of $\Delta$ acting on $\mathscr{H}_{(\ell)}$ (that is, the space of $L^{2}\left(\mathbb{R}^{3}\right)$ functions whose "angular momentum" is $\ell \geq 0$ ). From (B-1) and (B-2) we deduce that the integral kernel of $e^{t \Delta_{(\ell)}}$ acting on $L^{2}\left(\mathbb{R}_{+}, r^{2} d r\right)$ is given by

$$
e^{t \Delta(\ell)}(r, s)=\frac{1}{2 t} \sqrt{\frac{1}{r s}} e^{-\left(r^{2}+s^{2}\right) /(4 t)} I_{\ell+1 / 2}\left(\frac{r s}{2 t}\right) .
$$

An explicit integral representation for $I_{k}(z)$ shows that $I_{\ell+1 / 2}(z)>0$ for all $z>0$ and $\ell \geq 0$.

\section{Acknowledgments}

It is a pleasure to thank Joachim Krieger and Maciej Zworski for helpful discussions, as well as Mathieu Lewin for pointing out to results on the nonrelativistic limit for Dirac-Fock equations. The author is also indebted to Mohammed Lemou and Pierre Raphaël, who found a gap in the previous version of this paper. This work was partially supported by the National Science Foundation Grant DMS-0702492.

\section{References}

[Abou Salem 2007] W. K. Abou Salem, "Solitary wave dynamics in time-dependent potentials", 2007. arXiv 0707.0272 [Albert 1995] J. Albert, "Positivity properties and uniqueness of solitary wave solutions of the intermediate long-wave equation”, pp. 11-20 in Evolution equations (Baton Rouge, LA, 1992), edited by G. Ferreyra et al., Lecture Notes in Pure and Appl. Math. 168, Dekker, New York, 1995. MR 95i:35240

[Amick and Toland 1991] C. J. Amick and J. F. Toland, "Uniqueness and related analytic properties for the Benjamin-Ono equation-a nonlinear Neumann problem in the plane", Acta Math. 167:1-2 (1991), 107-126. MR 92i:35099 Zbl 0755.35108

[Bronski and Jerrard 2000] J. C. Bronski and R. L. Jerrard, "Soliton dynamics in a potential", Math. Res. Lett. 7:2-3 (2000), 329-342. MR 2001g:37139 Zbl 0955.35067 
[Carmona and Simon 1981] R. Carmona and B. Simon, "Pointwise bounds on eigenfunctions and wave packets in $N$-body quantum systems, V: Lower bounds and path integrals”, Comm. Math. Phys. 80:1 (1981), 59-98. MR 84m:81052a Zbl 0464. 35085

[Chang 2005] K.-C. Chang, Methods in nonlinear analysis, Springer Monographs in Mathematics, Springer, Berlin, 2005. MR 2007b:47169 Zbl 1081.47001

[Chang et al. 2007] S.-M. Chang, S. Gustafson, K. Nakanishi, and T.-P. Tsai, "Spectra of linearized operators for NLS solitary waves", SIAM J. Math. Anal. 39:4 (2007), 1070-1111. MR 2008k:35400 Zbl 05306587

[Cho and Ozawa 2006] Y. Cho and T. Ozawa, "On the semirelativistic Hartree-type equation”, SIAM J. Math. Anal. 38:4 (2006), 1060-1074. MR 2007m:35249 Zbl 1122.35119

[Elgart and Schlein 2007] A. Elgart and B. Schlein, "Mean field dynamics of boson stars", Comm. Pure Appl. Math. 60:4 (2007), 500-545. MR 2009a:85001 Zbl 1113.81032

[Esteban and Séré 2001] M. J. Esteban and E. Séré, "Nonrelativistic limit of the Dirac-Fock equations", Ann. Henri Poincaré 2:5 (2001), 941-961. MR 2003d:81066 Zbl 1092.81520

[Fröhlich and Lenzmann 2007] J. Fröhlich and E. Lenzmann, "Blowup for nonlinear wave equations describing boson stars", Comm. Pure Appl. Math. 60:11 (2007), 1691-1705. MR 2008m:85002 Zbl 1126.35064

[Fröhlich et al. 2002] J. Fröhlich, T.-P. Tsai, and H.-T. Yau, "On the point-particle (Newtonian) limit of the nonlinear Hartree equation”, Comm. Math. Phys. 225:2 (2002), 223-274. MR 2003e:81047 Zbl 1025.81015

[Fröhlich et al. 2004] J. Fröhlich, S. Gustafson, B. L. G. Jonsson, and I. M. Sigal, "Solitary wave dynamics in an external potential", Comm. Math. Phys. 250:3 (2004), 613-642. MR 2005h:35320 Zbl 1075.35075

[Fröhlich et al. 2007a] J. Fröhlich, B. L. G. Jonsson, and E. Lenzmann, "Boson stars as solitary waves", Comm. Math. Phys. 274:1 (2007), 1-30. MR 2008e:35183

[Fröhlich et al. 2007b] J. Fröhlich, B. L. G. Jonsson, and E. Lenzmann, "Effective dynamics for boson stars", Nonlinearity 20:5 (2007), 1031-1075. MR 2312382 Zbl 1124.35084

[Harrison et al. 2003] R. Harrison, I. Moroz, and K. P. Tod, "A numerical study of the Schrödinger-Newton equations", Nonlinearity 16:1 (2003), 101-122. MR 2004a:81082 Zbl 1040.81554

[Herbst 1977] I. W. Herbst, "Spectral theory of the operator $\left(p^{2}+m^{2}\right)^{1 / 2}-Z e^{2} / r$ ", Comm. Math. Phys. 53:3 (1977), $285-294$. MR 55 \#9790 Zbl 0375.35047

[Holmer and Zworski 2008] J. Holmer and M. Zworski, "Soliton interaction with slowly varying potentials", Int. Math. Res. Not. IMRN 10 (2008), Art. ID rnn026, 36. MR MR2429244 Zbl 1147.35084

[Jonsson et al. 2006] B. L. G. Jonsson, J. Fröhlich, S. Gustafson, and I. M. Sigal, "Long time motion of NLS solitary waves in a confining potential”, Ann. Henri Poincaré 7:4 (2006), 621-660. MR 2007f:35269 Zbl 1100.81019

[Krieger et al. 2008] J. Krieger, I. Martel, and P. Raphaël, "Two soliton solutions to the three dimensional gravitational Hartree equation”, Preprint, 2008.

[Kwong 1989] M. K. Kwong, "Uniqueness of positive solutions of $\Delta u-u+u^{p}=0$ in $\mathbf{R}^{n}$ ", Arch. Rational Mech. Anal. 105:3 (1989), 243-266. MR 90d:35015 Zbl 0676.35032

[Lenzmann 2006] E. Lenzmann, Nonlinear dispersive equations describing boson stars, Ph.D. dissertation No. 16572, ETH Zürich, 2006.

[Lenzmann 2007] E. Lenzmann, "Well-posedness for semirelativistic Hartree equations of critical type", Math. Phys. Anal. Geom. 10:1 (2007), 43-64. MR 2008i:35228

[Lieb 1977] E. H. Lieb, "Existence and uniqueness of the minimizing solution of Choquard's nonlinear equation", Studies in Appl. Math. 57:2 (1977), 93-105. MR 57 \#11508 Zbl 0369.35022

[Lieb and Loss 2001] E. H. Lieb and M. Loss, Analysis, Second ed., Graduate Studies in Math. 14, Amer. Math. Soc., Providence, RI, 2001. MR 2001i:00001 Zbl 0966.26002

[Lieb and Yau 1987] E. H. Lieb and H.-T. Yau, "The Chandrasekhar theory of stellar collapse as the limit of quantum mechanics", Comm. Math. Phys. 112:1 (1987), 147-174. MR 89b:82014 Zbl 0641.35065

[McLeod 1993] K. McLeod, "Uniqueness of positive radial solutions of $\Delta u+f(u)=0$ in $\mathbf{R}^{n}$. II", Trans. Amer. Math. Soc. 339:2 (1993), 495-505. MR 94b:35105 Zbl 0804.35034 
[McLeod and Serrin 1987] K. McLeod and J. Serrin, "Uniqueness of positive radial solutions of $\Delta u+f(u)=0$ in $\mathbf{R}^{n "}$, Arch. Rational Mech. Anal. 99:2 (1987), 115-145. MR 88c:35057 Zbl 0667.35023

[Peletier and Serrin 1983] L. A. Peletier and J. Serrin, "Uniqueness of positive solutions of semilinear equations in $\mathbf{R}^{n}$ ", $\operatorname{Arch}$. Rational Mech. Anal. 81:2 (1983), 181-197. MR 84b:35046 Zbl 0516.35031

[Reed and Simon 1978] M. Reed and B. Simon, Methods of modern mathematical physics, IV, Analysis of operators, Academic Press (Harcourt Brace Jovanovich), New York, 1978. MR 0493421 Zbl 0401.47001

[Reed and Simon 1980] M. Reed and B. Simon, Methods of modern mathematical physics, I, Functional analysis, Second ed., Academic Press (Harcourt Brace Jovanovich), New York, 1980. MR 85e:46002 Zbl 0459.46001

[Strauss 1977] W. A. Strauss, "Existence of solitary waves in higher dimensions", Comm. Math. Phys. 55:2 (1977), 149-162. MR 56 \#12616 Zbl 0356.35028

[Tod and Moroz 1999] P. Tod and I. M. Moroz, "An analytical approach to the Schrödinger-Newton equations", Nonlinearity 12:2 (1999), 201-216. MR 2000j:81053 Zbl 0942.35077

[Weinstein 1985] M. I. Weinstein, "Modulational stability of ground states of nonlinear Schrödinger equations", SIAM J. Math. Anal. 16:3 (1985), 472-491. MR 86i:35130 Zbl 0583.35028

Received 25 Jan 2008. Revised 17 Sep 2008. Accepted 11 Jan 2009.

ENNO LENZMANN: lenzmann@math.mit.edu

Massachusetts Institute of Technology, Department of Mathematics, Room 2-230, Cambridge, MA 02139, United States 


\title{
Analysis \& PDE
}

pjm.math.berkeley.edu/apde

EDITORS

\author{
EDITOR-IN-CHIEF \\ Maciej Zworski \\ University of California \\ Berkeley, USA
}

BOARD OF EDITORS

\begin{tabular}{|c|c|c|c|}
\hline Michael Aizenman & $\begin{array}{l}\text { Princeton University, USA } \\
\text { aizenman@math.princeton.edu }\end{array}$ & Nicolas Burq & $\begin{array}{l}\text { Université Paris-Sud 11, France } \\
\text { nicolas.burq@math.u-psud.fr }\end{array}$ \\
\hline Luis A. Caffarelli & $\begin{array}{l}\text { University of Texas, USA } \\
\text { caffarel@math.utexas.edu }\end{array}$ & un-Yung Alice Chang & $\begin{array}{l}\text { Princeton University, USA } \\
\text { chang@math.princeton.edu }\end{array}$ \\
\hline Michael Christ & $\begin{array}{l}\text { University of California, Berkeley, USA } \\
\text { mchrist@math.berkeley.edu }\end{array}$ & Charles Fefferman & $\begin{array}{l}\text { Princeton University, USA } \\
\text { cf@math.princeton.edu }\end{array}$ \\
\hline Ursula Hamenstaedt & $\begin{array}{l}\text { Universität Bonn, Germany } \\
\text { ursula@math.uni-bonn.de }\end{array}$ & Nigel Higson & $\begin{array}{l}\text { Pennsylvania State Univesity, USA } \\
\text { higson@math.psu.edu }\end{array}$ \\
\hline Vaughan Jones & $\begin{array}{l}\text { University of California, Berkeley, USA } \\
\text { vfr@math.berkeley.edu }\end{array}$ & Herbert Koch & $\begin{array}{l}\text { Universität Bonn, Germany } \\
\text { koch@math.uni-bonn.de }\end{array}$ \\
\hline Izabella Laba & $\begin{array}{l}\text { University of British Columbia, Canada } \\
\text { ilaba@math.ubc.ca }\end{array}$ & Gilles Lebeau & $\begin{array}{l}\text { Université de Nice Sophia Antipolis, France } \\
\text { lebeau@unice.fr }\end{array}$ \\
\hline László Lempert & $\begin{array}{l}\text { Purdue University, USA } \\
\text { lempert@math.purdue.edu }\end{array}$ & Richard B. Melrose & $\begin{array}{l}\text { Massachussets Institute of Technology, USA } \\
\text { rbm@math.mit.edu }\end{array}$ \\
\hline Frank Merle & $\begin{array}{l}\text { Université de Cergy-Pontoise, France } \\
\text { Frank.Merle@u-cergy.fr }\end{array}$ & William Minicozzi II & $\begin{array}{l}\text { Johns Hopkins University, USA } \\
\text { minicozz@math.jhu.edu }\end{array}$ \\
\hline Werner Müller & $\begin{array}{l}\text { Universität Bonn, Germany } \\
\text { mueller@math.uni-bonn.de }\end{array}$ & Yuval Peres & $\begin{array}{l}\text { University of California, Berkeley, USA } \\
\text { peres@stat.berkeley.edu }\end{array}$ \\
\hline Gilles Pisier & $\begin{array}{l}\text { Texas A\&M University, and Paris } 6 \\
\text { pisier@math.tamu.edu }\end{array}$ & Tristan Rivière & $\begin{array}{l}\text { ETH, Switzerland } \\
\text { riviere@math.ethz.ch }\end{array}$ \\
\hline Igor Rodnianski & $\begin{array}{l}\text { Princeton University, USA } \\
\text { irod@ math.princeton.edu }\end{array}$ & Wilhelm Schlag & $\begin{array}{l}\text { University of Chicago, USA } \\
\text { schlag@ math.uchicago.edu }\end{array}$ \\
\hline Sylvia Serfaty & $\begin{array}{l}\text { New York University, USA } \\
\text { serfaty@ cims.nyu.edu }\end{array}$ & Yum-Tong Siu & $\begin{array}{l}\text { Harvard University, USA } \\
\text { siu@math.harvard.edu }\end{array}$ \\
\hline Terence Tao & $\begin{array}{l}\text { University of California, Los Angeles, USA } \\
\text { tao@math.ucla.edu }\end{array}$ & Michael E. Taylor & $\begin{array}{l}\text { Univ. of North Carolina, Chapel Hill, USA } \\
\text { met@ math.unc.edu }\end{array}$ \\
\hline Gunther Uhlmann & $\begin{array}{l}\text { University of Washington, USA } \\
\text { gunther@math.washington.edu }\end{array}$ & András Vasy & $\begin{array}{l}\text { Stanford University, USA } \\
\text { andras@math.stanford.edu }\end{array}$ \\
\hline an Virgil Voiculescu & $\begin{array}{l}\text { University of California, Berkeley, USA } \\
\text { dvv@ math.berkeley.edu }\end{array}$ & Steven Zelditch & $\begin{array}{l}\text { Johns Hopkins University, USA } \\
\text { szelditch@math.jhu.edu }\end{array}$ \\
\hline
\end{tabular}

\section{PRODUCTION}

apde@mathscipub.org

Paulo Ney de Souza, Production Manager Sheila Newbery, Production Edito

Silvio Levy, Senior Production Editor

See inside back cover or pjm.math.berkeley.edu/apde for submission instructions.

The subscription price for 2009 is US \$120/year for the electronic version, and \$180/year for print and electronic. Subscriptions, requests for back issues from the last three years and changes of subscribers address should be sent to Mathematical Sciences Publishers, Department of Mathematics, University of California, Berkeley, CA 94720-3840, USA.

Analysis \& PDE, at Mathematical Sciences Publishers, Department of Mathematics, University of California, Berkeley, CA 94720-3840 is published continuously online. Periodical rate postage paid at Berkeley, CA 94704, and additional mailing offices.

APDE peer-review and production is managed by EditFLOW ${ }^{\mathrm{TM}}$ from Mathematical Sciences Publishers.

PUBLISHED BY

mathematical sciences publishers

http://www.mathscipub.org

A NON-PROFIT CORPORATION

Typeset in IATEX

Copyright (C2009 by Mathematical Sciences Publishers 


\section{ANALYSIS \& PDE}

\section{Volume 2 No. $1 \quad 2009$}

Uniqueness of ground states for pseudorelativistic Hartree equations

ENNO LENZMANN

Resonances for nonanalytic potentials

ANDrÉ MARTINEZ, THIERRY RAMOND and JOHANNES SJÖSTRAND

Global existence and uniqueness results for weak solutions of the focusing mass-critical nonlinear Schrödinger equation

TERENCE TAO

The linear profile decomposition for the Airy equation and the existence of maximizers for the Airy Strichartz inequality

SHUANGLIN SHAO 